\title{
ИНТЕГРАЦИЯ ПРОЦЕССА КАТЕГОРИЗАЦИИ ЭЛЕКРОХИМИЧЕСКИХ НАКОПИТЕЛЕЙ ЭНЕРГИИ В ЗАДАЧУ ОПТИМИЗАЦИИ СОСТАВА ОБОРУДОВАНИЯ АВТОНОМНЫХ ЭНЕРГЕТИЧЕСКИХ КОМПЛЕКСОВ, ИСПОЛЬЗУЮЩИХ ВОЗОБНОВЛЯЕМЫЕ ИСТОЧНИКИ ЭНЕРГИИ
}

\author{
Карамов Дмитрий Николаевич,2, \\ dmitriy.karamov@mail.ru \\ 1 Институт систем энергетики им. Л.А. Мелентьева Сибирского отделения Российской академии наук, \\ Россия, 664033, г. Иркутск, ул. Лермонтова, 130. \\ 2 Иркутский национальный исследовательский технический университет, \\ Россия, 664074, г. Иркутск, ул. Лермонтова, 83.
}

\begin{abstract}
Актуальность работы обусловлена необходимостью повышения точности решения задачи оптимизации состава оборудования автономных энергетических комплексов, использующих возобновляемые источники и накопители энергии

Цель: продемонстрировать возможность интеграции процесса категоризации электрохимических накопителей энергии в задачу оптимизации состава оборудования автономных энергетических комплексов, использующих возобновляемые источники и накопители энергии; выявить ряд закономерностей, влияющих на процесс категоризации электрохимических накопителей энергии, с последующим обобщением и детальным анализом полученной информации.

Методы. Представленная методика последовательно выстроена на положениях теории систем с использованием общеизвестных математических моделей, позволяющих моделировать основные режимные параметры автономных систем электроснабжения с возобновляемыми источниками и электрохимическими накопителями энергии. Используются актинометрические, ветроэнергетические и многие другие природно-климатические показатели, полученные в результате обработки многолетних метеорологических рядов, зафиксированных на метеостанциях Ном, Кодиак, Адак (штат Аляска, США).

Результаты. Предложена методика интеграции процесса категоризации электрохимических накопителей энергии в задачу оптимизации состава оборудования автономных систем электроснабжения, использующих возобновляемые источники и накопители энергии. Представлены результаты исследования трех автономных энергетических комплексов, использующих возобновляемые источники и накопители энергии. По итогам исследования были получены результаты, характеризующие зависимость между составом генерирующего оборудования и суммарной установленной емкостью аккумулирующего звена. Проведен детальный анализ полученных результатов с соответствующими выводами, визуализацией и заключениями о степени их достоверности и возможности использования в оптимизационных исследованиях автономных энергетических комплексов, использующих возобновляемые источники и накопители энергии.
\end{abstract}

\section{Ключевые слова:}

Возобновляемые источники энергии, категоризация аккумуляторных батарей,

деградация аккумуляторных батарей, системные исследования, оптимизация состава оборудования.

\section{Введение}

На протяжении последнего десятилетия мировой опыт показывает, что комбинированное использование возобновляемых источников энергии (ВИЭ) совместно с традиционными энергетическими установками и электрохимическими накопителями электрической энергии является экономически эффективным способом энергообеспечения потребителей в автономных системах электроснабжения (АСЭС) [1-5]. При этом особую значимость и большое распространение ВИЭ получили при электрификации труднодоступных регионов мира, не имеющих связи с традиционными централизованными энергетическими системами.

Стоит отметить, что одной из главных задач при использовании ВИЭ и электрохимических накопителей энергии в АСЭС являются задачи, отвечающие за нахождение оптимальных технологий производства, передачи, трансформации и аккумулирования энергии с последующим определением необходимых значений установленных мощностей основного генерирующего оборудования, элементов электрической, распределительной сети и суммарной емкости накопителей энергии [6-12]. Таким образом, задачи оптимизации позволяют определить такой состав оборудования, при котором достигаются минимальные значения целевой функции, которая, как правило, представлена нормированной стоилостью электроэнергии (levelized cost of electricity - LCOE) [12-15]. LCOE - хорошо зарекомендовала себя при решении задачи оптимизации состава оборудования и реализована во многих программно-вычислительных комплексах (ПВК), имеющих большое распространение как с научной, так и практической точки зрения.

К таким ПВК относятся HOGA - Hybrid optimization by genesis algorithm (Испания), HOMER Hybrid optimization modeling software (CШA), HYPORA - Hybrid power optimized for rural/remote areas (CШA), TRNSYS - Transient system simulation tool (США) и т. д. [12, 16]. В данных ПВК используются различные подходы к анализу природно-климатических показателей, разные по уровню детализации модели элементов генерации, силовой электроники, аккумулирующих устройств и других элементов энергетической системы, объединенных единым процессом производства, передачи, аккумулирования энергии. Стоит отметить, что в данных ПВК нашли применение разнообразные методы оптимизации [16. С. 20]. 
При этом важно отметить, что конечный результат оптимизации напрямую зависит от того, какие технологии производства, передачи, трансформации и аккумулирования электрической энергии рассматривались в рамках исследования [17, 18]. Другими словами, оптимизация состава оборудования зависит от первоначальной матрицы расслатриваемого оборудования, в рамках которой находятся потенциально возможные технологии с подробным техническим и экономическим описанием линейки типоразмеров. Следовательно, рассмотрение различных колбинаций состава оборудования в рамках решения задачи оптимизации позволяет определить как наиболее эффективные технологии производства, трансформации, передачи и аккумулирования электрической энергии, так и оптимальную установленную мощность оборудования из рассматриваемой линейки типоразмеров $[19,20]$.

Стоит упомянуть, что элементы аккумулирования, электрической энергии, которые, как правило, представлены разного рода аккумуляторными батареями (АБ) в отличие от элементов генерации и трансформации электрической энергии имеют определенный ряд специфических особенностей, которые существенно влияют на конечный результат оптимизации [21].

К данным особенностям относятся внутренние процессы старения активной массы АБ, включая расслоение электролита, разрушение положительного электрода, тяжелую/необратимую сульфацию, кристаллизацию и т. д., исходя из условий их эксплуатации [21-23]. При этом важно отметить, что данные положения в большей степени относятся к химическому направлению, что существенно усложняет процесс моделирования работы АБ с учетом вышеупомянутых факторов. Данные факторы имеют совершенно разную частоту возникновения в зависимости от рассматриваемого типа, установленной ёмкости АБ и условий её эксплуатации [23]. В добавок следует отметить, что в рамках задачи оптимизации, где процесс поиска минимума (или максимума) целевой функции зачастую является циклическим много итерационным, что существенно усложняет использование ресурсоемких моделей АБ. Кроме того, данные процессы еще не до конца изучены и требуют более детальной проработки химической составляющей, отвечающей за деградацию активной массы АБ с последующей разработкой агрегированных моделей учета данных процессов.

Учет вышеупомянутых положений позволяет выработать ряд мероприятий по выбору наиболее оптимальной технологии аккумулирования энергии в зависимости от условий эксплуатации АБ и ряда других режимных параметров, а также сократить количество вариантов потенциально возможных составов оборудования.

\section{Роль аккумуляторных батарей в АСЭС}

В целом структура АСЭС может быть описана с помощью ориентированного графа, в котором все эле- менты и связи между ними обозначены цифрами. В таком случае определяется суммарное количество электрических связей $n$, а также число узлов схемы $m$, которые представлены элементами генерации и аккумулирования энергии с последующим обозначением линейно независимых контуров [24]. На рис. 1 показан ориентированный граф АСЭС с ВИЭ и АБ.

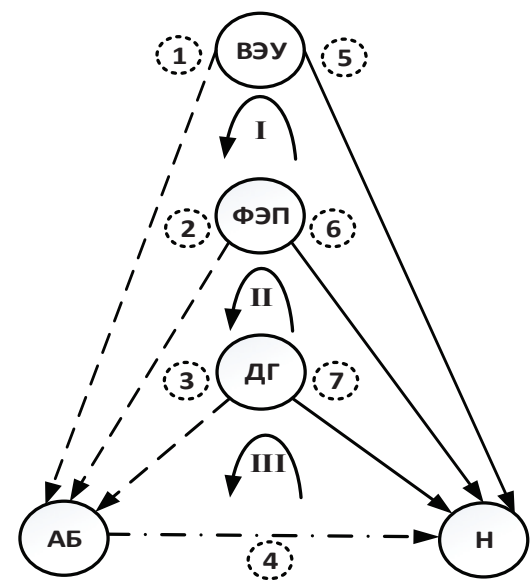

Pис. 1. Ориентированный граф АСЭС с ВИЭ и АБ: ВЭУ - ветроэнергетическая установка; ФЭП - фотоэлектрические преобразователи; ДГ - дизель генератор; АБ -аккулуляторные батареи; $\mathrm{H}$-нагрузка

Fig. 1. Oriented graph of autonomous power supply system, using renewable energy sources and storage batteries: ВЭУ - wind turbine; ФЭП - photovoltaics; ДГ - diesel generator; $А Б$ storage batteries; $\mathrm{H}$ - electrical load

Представленный на рис. 1 ориентированный граф АСЭС имеет пять основных узлов, которые представлены элементами генерации (ВЭУ, ФЭП, $Д \Gamma)$, электрической нагрузкой $(H)$, аккумуляторными батареями $(A \overline{)})$, а также селью электрическими связями и 3 линейно независимыми контурами. При этом четыре из пяти узлов являются линейно независимыми. Дальнейший анализ представленного ориентированного графа позволяет составить две матрицы: $A$ - матрица соединений линейно независимых узлов и участков ориентированного графа размерностью $(m-1) \times n$ и $B-$ матрица линейно независимых контуров и участков цепи, размерностью $c \times n$, где $c=n-(m-1)$. Из ориентированного графа следует, что известными параметрами системы являются значения генерируемой мощности в узлах системы, т. е. векторфункции скалярного аргумента $t$.

Матрица $A$ формируется исходя из следующего правила: если $j$-му узлу принадлежит $i$-й участок и направление его из узла, то элемент матрицы равен -1 , если $j$-му узлу принадлежит $i$-й участок и направление его в узел, то элемент матрицы равен +1 , иначе элемент матрицы равен 0 , тогда:

$$
A=\left|\begin{array}{c}
\rightleftarrows \\
\text { ВЭУ } \\
\text { ФЭП } \\
\text { ДГ } \\
\text { АБ }
\end{array}\right|\left[\begin{array}{ccccccc}
\underline{1} & \underline{2} & \underline{3} & \underline{4} & \underline{5} & \underline{6} & \underline{7} \\
-1 & 0 & 0 & 0 & -1 & 0 & 0 \\
0 & -1 & 0 & 0 & 0 & -1 & 0 \\
0 & 0 & -1 & 0 & 0 & 0 & -1 \\
0 & 0 & 0 & -1 & 1 & 1 & 1
\end{array}\right]
$$


Элементы матрицы постоянные численные величины и элементы для узла $H$ могут быть получены в результате суммирования по столбцам, умноженным на множитель -1 . Таким образом, можно записать матрицу $A$ в виде блочных матриц $A=\left[A_{d} ; A_{c}\right]$,

$$
A_{d}=\left|\begin{array}{c}
\rightleftarrows \\
\text { ВЭУ } \\
\text { ФП } \\
\text { ДГ } \\
\text { АБ }
\end{array}\right|\left[\begin{array}{cccc}
\underline{1} & \underline{2} & \underline{3} & 4 \\
-1 & 0 & 0 & 0 \\
0 & -1 & 0 & 0 \\
0 & 0 & -1 & 0 \\
0 & 0 & 0 & -1
\end{array}\right], \quad A_{c}=\left|\begin{array}{c}
\rightleftarrows \\
\text { ВЭУ } \\
\text { ФЭП } \\
\text { ДГ } \\
\text { АБ }
\end{array}\right|\left[\begin{array}{ccc}
\underline{5} & \underline{6} & \underline{7} \\
-1 & 0 & 0 \\
0 & -1 & 0 \\
0 & 0 & -1 \\
1 & 1 & 1
\end{array}\right] .
$$

Матрица $B$ образуется согласно следующему правилу: если $c$-му контуру принадлежит $i$-й участок и направление его совпадает с направлением контура, то элемент матрицы равен +1 , если $c$-му узлу принадлежит $i$-й участок и направление его противоположное направлению контура, то элемент матрицы равен - 1 , иначе элемент матрицы равен 0, следовательно:

$$
B=\left|\begin{array}{l}
\leftrightarrows \\
I I \\
I I \\
I I I
\end{array}\right|\left[\begin{array}{ccccccc}
\underline{1} & \underline{2} & \underline{3} & \underline{4} & \underline{5} & \underline{6} & \underline{7} \\
-1 & 0 & 0 & 1 & 1 & 0 & 0 \\
0 & -1 & 0 & 1 & 0 & 1 & 0 \\
0 & 0 & -1 & 1 & 0 & 0 & 1
\end{array}\right] .
$$

Матрицу линейно независимых контуров и участков системы также можно представить в блочном виде $B=\left[B_{d} ; B_{c}\right]$.

$$
B_{d}=\left|\begin{array}{c}
\leftrightarrows \\
I \\
I I \\
I I I
\end{array}\right|\left[\begin{array}{cccc}
\underline{1} & \underline{2} & \underline{3} & \underline{4} \\
-1 & 0 & 0 & 1 \\
0 & -1 & 0 & 1 \\
0 & 0 & -1 & 1
\end{array}\right], \quad B_{c}=\left|\begin{array}{c}
\leftrightarrows \\
I I \\
I I I
\end{array}\right| \mid\left[\begin{array}{ccc}
\underline{5} & \underline{6} & \frac{7}{1} \\
1 & 0 & 0 \\
0 & 1 & 0 \\
0 & 0 & 1
\end{array}\right] .
$$

Анализ матриц $A_{d}, A_{c}, B_{d}, B_{c}$ позволяет сделать вывод, что узел $A Б$ имеет максимальную степень среди остальных узлов ориентированного графа. При этом узел $A Б$ имеет непосредственную связь с узлом нагрузки $H$ в моменты, когда

$$
P_{S}(t)<0 \text {, }
$$

где

$$
\begin{gathered}
P_{S}(t)=P_{\text {вэу }}(t)+P_{\text {Фэп }}(t)+P_{\text {дГ }}(t)-P_{H}(t)-\Delta P_{\Sigma}(t), \\
\Delta P_{\Sigma}(t)=\Delta P_{\text {Кл-вл }}(t)+\Delta P_{\text {Син }}+\Delta P_{\text {Бин }}(t)+\Delta P_{\text {АБ }}(t),
\end{gathered}
$$

где $P_{\text {дг }}(t)$ - генерация ДГ в момент, когда состояние заряда имеет минимально-допустимое значение. При этом ДГ включается на полную мощность, осуществляя прямое снабжение потребителя и заряд АБ. В таких условиях ДГ имеет минимальный удельный расход дизельного топлива.

Следовательно, исходя из анализа матриц $A$ и $B$, а также основных уравнений, описывающих знакопереленную функцию мощности $P_{S}(t)$, можно сделать вывод, что узел $A Б$ в АСЭС с ВИЭ является стабилизирующим элементом системы, работающим в буферном режиме, который позволяет уменьшить влияние разного рода и уровня возмущений в энергетической системе [25-28].

\section{Аккумуляторные батареи в АСЭС с ВИЭ}

Работа АБ в АСЭС с ВИЭ имеет ряд особенностей, влияющих как на техническую, так и на экономическую эффективность использования тех или иных типов электрохимических накопителей энергии [16].

В первую очередь данные особенности в большей степени отражены в неравномерности выработки электрической энергии генераторами, использующими в качестве первичных двигателей энергию ветра и Солнца $[20,21]$. Стохастическая составляющая при генерировании электрической энергии существенно сказывается на основных эксплуатационных показателях АБ. К примеру, в системах электроснабжения, где в качестве основного генерирующего оборудования используются ФЭП, эксплуатация АБ сопровождается процессами с ярко выраженным циклическим характером в течение суток. На рис. 2 показано А - изменение состояния заряда АБ и В - поведение знакопеременной функции мощности системы на протяжении четырех летних дней в АСЭС с ФЭП и АБ.

Как видно из рис. 2 , состояние заряда АБ можно разделить на три условных режима.

1. Заряд АБ в момент, когда знакопеременная функция имеет положительный знак:

$$
P_{S}(t)^{+}=\left\{\begin{array}{c}
P_{S}(t) ;\left(P_{S}(t)>0\right) \wedge\left(\bar{a}_{\mathrm{AB}}\right) \geq \bar{b}_{\mathrm{AB}} \cdot P_{S}(t) \cdot \Delta t \\
\frac{\left(\bar{a}_{\mathrm{A} A}\right)}{\Delta t} ;\left(P_{S}(t)>0\right) \wedge\left(\bar{a}_{\mathrm{AB}}\right)<\bar{b}_{\mathrm{AB}} \cdot P_{S}(t) \cdot \Delta t \\
0 ; P_{S}(t)<0
\end{array}\right\} .
$$

2. Разряд АБ в момент, когда знакопеременная функция имеет отрицательный знак:

$$
P_{S}(t)^{-}=\left\{\begin{array}{c}
P_{S}(t) ;\left(P_{S}(t)<0\right) \wedge\left(\bar{a}_{\mathrm{AB}}\right) \geq \frac{P_{S}(t)}{\bar{b}_{\mathrm{AB}}} \cdot \Delta t \\
\frac{\left(\bar{a}_{\mathrm{AB}}\right)}{\bar{b}_{\mathrm{AB}}} \cdot \Delta t ;\left(P_{S}(t)<0\right) \wedge\left(\bar{a}_{\mathrm{AB}}\right)<\frac{P_{S}(t)}{\bar{b}_{\mathrm{AB}}} \cdot \Delta t \\
0 ; P_{S}(t)>0
\end{array}\right\},
$$

3. Прямое снабжение потребителя от ВИЭ в момент, когда АБ заряжены на $100 \%$ :

$$
P_{S}(t)^{0}=\left\{\begin{array}{c}
P_{H}(t) ;\left(P_{S}(t)>P_{H}(t)\right) \wedge\left(Q_{\mathrm{AB}}(t)\right)=100 \\
0 ;\left(Q_{\mathrm{AB}}(t)\right)<100
\end{array}\right\},
$$

где $\bar{a}_{\mathrm{AБ}}$ - система ограничений по величинам заряда и разряда АБ, основанная на технических характеристиках, которая определяется следующим образом:

$$
\bar{a}_{\mathrm{AB}}=\left(Q_{\mathrm{AB}}^{\max }-Q_{\mathrm{AB}}^{\min }\right) \cdot \bar{k}_{\mathrm{AB}},
$$

где $Q_{\mathrm{Ab}}^{\max }$ - установленная емкость АБ, кВт•ч; $Q_{\mathrm{Ab}}^{\min }-$ минимально допустимое состояние заряда АБ, при котором включается резервный источник (как правило, составляет от 20 до $30 \%$ от $\left.Q_{\mathrm{AB}}^{\max }\right)$, кВт•ч; 


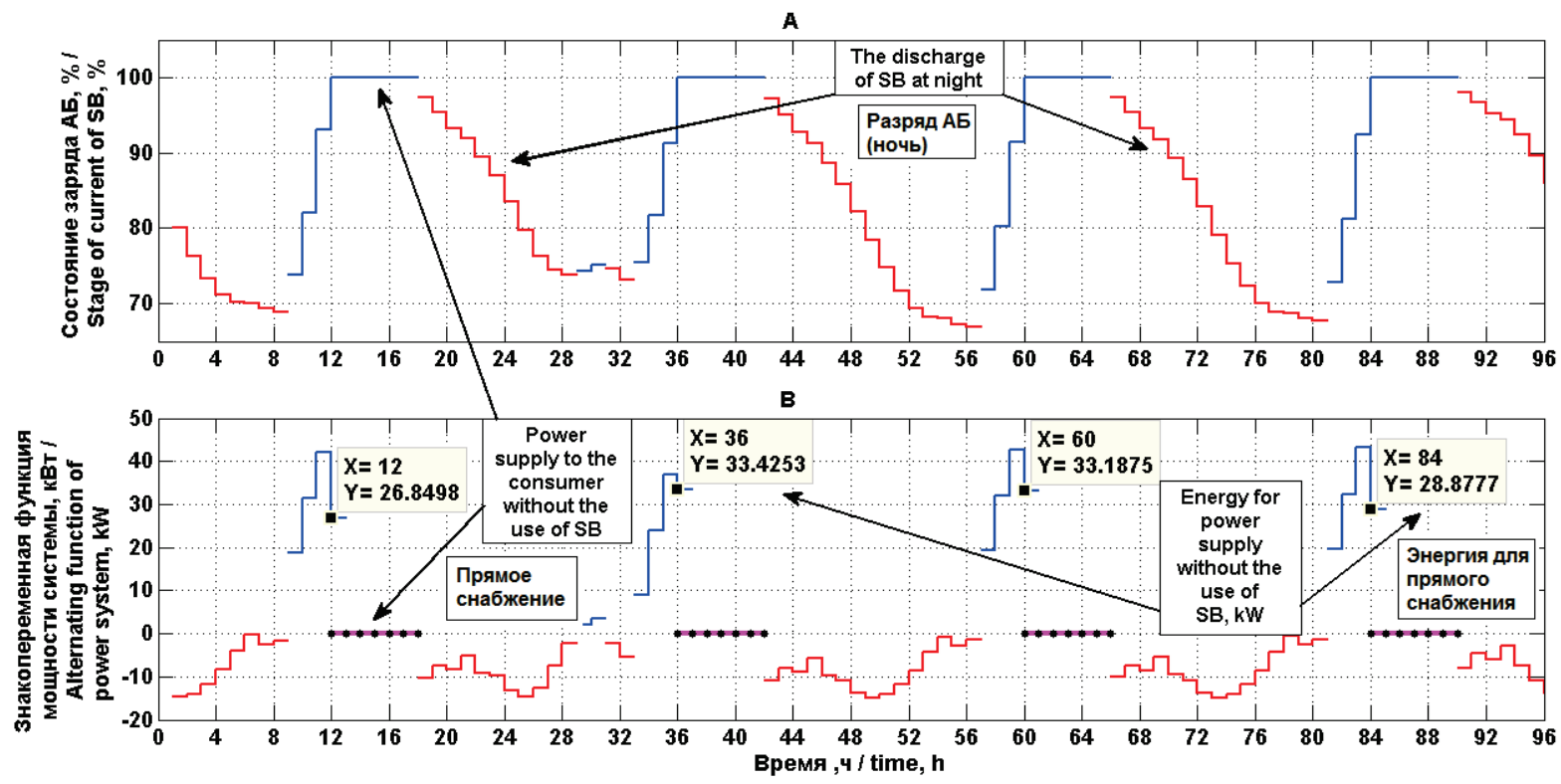

* SB - storage batteries

Pис. 2. А) изменение состояния заряда АБ; В) поведение знакопеременной функиии мощности системы на протяжении четырех летних дней в АСЭС с ФЭП и АБ

Fig. 2. A) stage of current of storage batteries; B) alternating function of power system during four summer days in the autonomous power supply system with photovoltaic panels and storage batteries

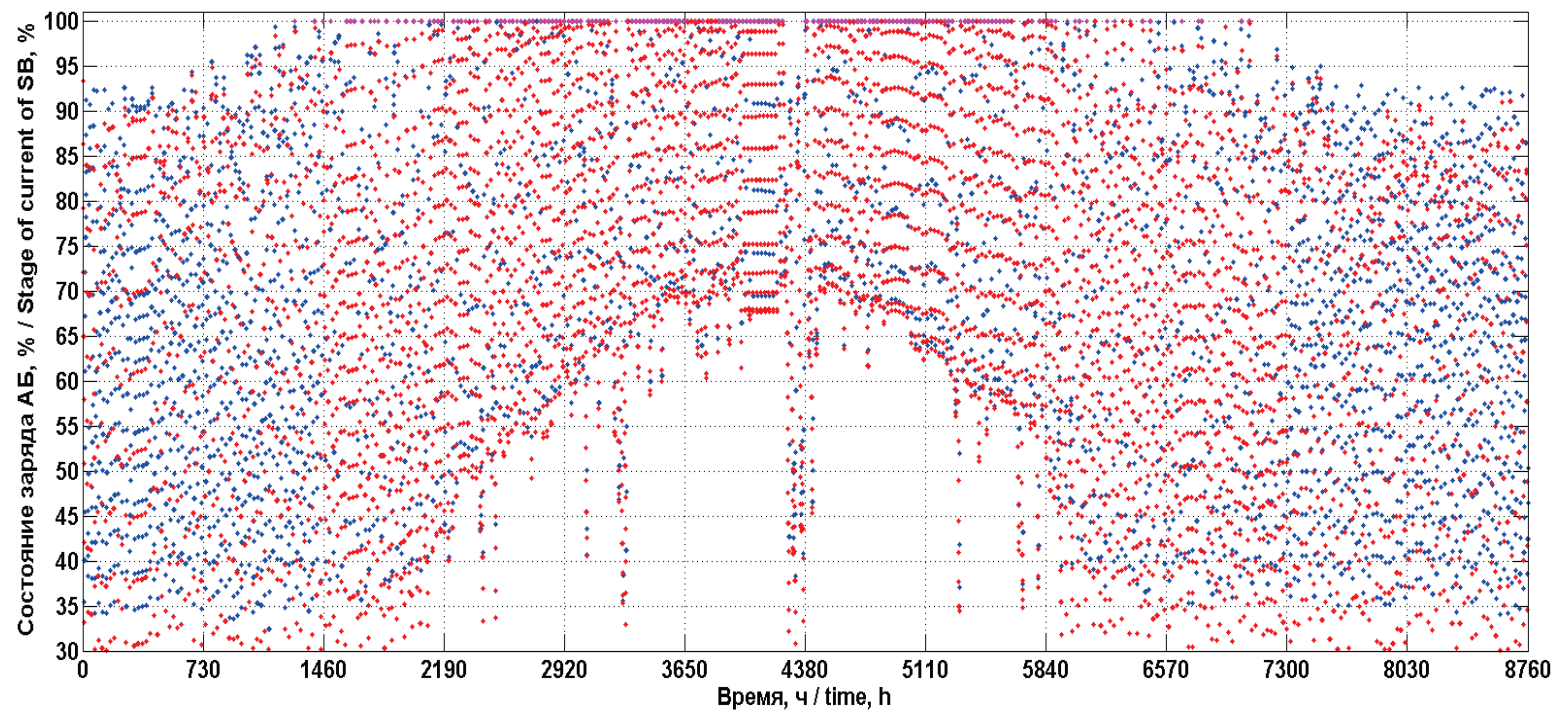

* Цвета/colors: красный/red - разряд/discharge; синий/bluе - заряд/charge;

розовый/pink - снабжение от ВИЭ без использования АБ/energy for power supply without the use of SB

Рис. 3. Изменение состояния заряда АБ в течение года в АСЭС с ФэП

Fig. 3. Stage of current of $S B$ during a year in autonomous power supply using photovoltaic panels

$\bar{k}_{\mathrm{AБ}}-$ заряд/разрядный коэффициент АБ (для разных типов АБ составляет от 10 до $40 \%$ от $\left.Q_{\mathrm{Ab}}^{\max }\right)$, $\kappa В T^{*} \div$.

Также составляется система логических условий преобразовательно-аккумулирующего звена $\bar{b}_{\mathrm{AБ}}$, которая выглядит следующим образом:

$$
\bar{b}_{\mathrm{AБ}}=\left\{\begin{array}{c}
\eta_{\mathrm{AБ}} \cdot \eta_{\text {Бин }} ;\left(P_{S}(t) \geq 0\right) \\
\eta_{\text {Бин }} ;\left(P_{S}(t)<0\right)
\end{array}\right\},
$$

$\eta_{\text {АБ }}, \eta_{\text {Бин }}-$ КПД АБ и батарейного инвертера, $\%$.

На рис. 2 показано изменение состояния заряда АБ в АСЭС с ФЭП в течение года с учетом вышеописанных логических условий.

Исходя из рис. 2, 3 значения знакопеременной функции мощности $P_{S}(t)$ относительно временного интервала $(t)$ описываются достаточно сложной системой логических условий. В рамках данных условий учитываются не только ряд эксплуата- 
ционно-технических ограничений, но и изменчивость природно-климатических показателей, влияющих на генерацию ФЭП и ВЭУ, что, несомненно, влияет на суммарное значение $P_{S}(t)$. С более подробным математическим описанием заряд/разрядных процессов различных видов АБ с увязкой системы управления резервных источников питания можно ознакомиться в [16. С. 44-51].

Негативные воздействия на аккумуляторные батареи

Работа АБ в АСЭС с ВИЭ зачастую сопровождается сложными эксплуатационными процессами, связанными с частыми циклическими разрядами на большую глубину АБ с последующим зарядом большими токами [29]. Частый неполный заряд с последующим разрядом до минимально допустимого состояния заряда $Q_{\mathrm{Ab}}^{\text {min }} \mathrm{AБ}$ приводит к процессу коррозии и разрушению положительных пластин, что ведет к выпадению активной массы. Таким образом, коррозия в АБ характеризуется нарастанием слоя оксида свинца на положительных пластинах с последующим их разрушением. Данный процесс приводит к снижению проводимости пластины, уменьшению её диаметра и увеличению сопротивления, что, несомненно, сказывается на суммарной располагаемой емкости АБ.

Также одним из процессов, ведущих к деградации активной массы АБ, является кислотная стратификация, которая характеризуется расслоением электролита на две составляющие. В таком случае кислота концентрируется в нижней части АБ и не в полном объёме взаимодействует с пластинами. Сама по себе кислотная стратификация не является процессом старения АБ. Однако она существенно влияет на газообразование, которое в активной массе ведет к смещению электролита и неравномерному распределению тока по пластинам, тем самым существенно ускоряя необратимый процесс сульфации и кристаллизации в нижней части АБ [30]. К техническим решениям, уменьшающим воздействие кислотной стратификации, относятся системы взбалтывания электролита. Также возможным решением является использование АБ технологии VRLA (valve-regulated lead-acid), в которой процесс кислотной стратификации практически исключен [30]. Особенность технологии $V R L A$ заключается в использовании не жидкого электролита, а абсорбированного (AGM - Absorbent Glass Mat) или гелевого (GB - gel battery).

Еще одним из важных процессов, существенно снижающих эксплуатационные показатели АБ, является сульфация, которая характеризуется уменьшением располагаемой емкости АБ, увеличением внутренней температуры АБ за счет увеличенного сопротивления пластин, существенным уменьшением плотности электролита, а также ранним газовыделением при заряде, что приводит к ошибочному прекращению заряда АБ и, как следствие, к частым недозарядам [31]. В конечном итоге процесс сульфации, несомненно, ведет к разрушению положительных пластин АБ. Основной причиной, ускоряющей процесс сульфации с последующей кристаллизацией, является частые глубокие разряды с длительным простоем в области пониженного заряда, близкого к минимально допустимому состоянию $Q_{\mathrm{AB}}^{\text {min. }}$. Следовательно, нахождение состояния заряда АБ в области минимально допустимых значений приводит к тому, что небольшие кристаллы сульфата свинца становятся опорныли центрами кристаллизации, что приводит к образованию крупнокристаллической формы сульфата и необратимой реакции [31, 32]. Также одной из причин повышенной сульфации является как существенно повышенная, так и пониженная температура окружающей среды. При этом в случае отрицательной температуры и замерзания электролита негативные воздействия в целом на АБ существенно возрастают и запускается необратилый процесс разрушения пластин и деградации всей активной массы АБ.

Таким образом, имеется целый ряд разного рода негативных воздействий на активную массу АБ, которые существенно снижают не только основные эксплуатационные параметры АБ, а в целом существенно сокращают располагаемый ресурс аккумулирующего звена. Естественно, важную роль в снижении негативных последствий для АБ играет правильно выстроенная система управления заряда АБ в моменты, когда $Q_{\mathrm{AB}}(t) \approx Q_{\mathrm{AB}}^{\min }$. Например, использование резервного источника питания для необходимого своевременного заряда АБ. Также немаловажную роль играют внешние условия эксплуатации, такие как автоматическое поддержание температурного режима в помещении (аккумуляторной) и т. д.

\section{Категоризация электрохимических накопителей энергии}

Первоначально процесс категоризации подразумевает детальный анализ химических, механических, электрических и пр. процессов, протекающих при эксплуатации АБ в системах электроснабжения, использующих ВИЭ, с последующим составлением категорий. Данные категории формируются вследствие анализа статистических данных об эксплуатационных режимах АБ в реальных АСЭС с ВИЭ. Кроме того, отдельные категории были получены в результате продолжительных по времени исследований ведущих европейских коллективов [32]. К примеру, в работах [29-32] приведен подробный анализ применения свинцово-кислотных АБ (AGM и $G B)$ в системах электроснабжения, использующих ФЭП. Представлены теоретические выкладки процессов старения и деградации активной массы АБ с последующей проверкой полученных результатов на реальных АСЭС. При этом даны основные рекомендации по процессам заряда и разряда АБ, потери части располагаемой емкости и возможные пути решения проблем деградации АБ.

Стоит отметить, что работы [29-32] не включали полный диапазон использования ВИЭ, в первую 
очередь ВЭУ, а также небольших энергетических систем. В связи с этим в 2007 г. был начат объединенный проект «Development of test procedures for benchmarking components in renewable energy systems applications, in particular energy storage systems», поддержанный Европейским Союзом, Правительством Австралии, Соединенных Штатов Америки и частными организациями. Данный проект позволил объединить ведущих мировых ученых в области аккумулирования энергии и других смежных направлений, связанных с решением ряда задач, возникающих в АСЭС с ВИЭ и АБ.

В рамках данного проекта была разработана достаточно интересная с практической и научной точки зрения методика категоризации АБ исходя из условий их эксплуатации. Было предложено шесть основных показателей, численный анализ которых позволяет с достаточной точностью описать процессы деградации активной массы АБ при имеющихся условиях эксплуатации. К данным факторам относятся:

Среднегодовой коэффиииент заряда - выражается средним коэффициентом заряда в рассматриваемый период времени. Данный показатель косвенно указывает, в каком режиме (плавающем заряде или циклическими разрядами) эксплуатируется АБ. В [23] отмечается, что в реальных системах с ВИЭ, как правило, имеют место оба из представленных режимов в зависимости уровня генерации ФЭП и ВЭУ и уровня нагрузки потребителя. Таким образом, коэффициент заряда может существенно варьироваться. Поэтому важно использовать данный показатель с остальными

$$
K_{\mathrm{Ab}}^{3}=\frac{\int_{1}^{\widehat{T}} I_{\mathrm{Ab}}(t) H\left(I_{\mathrm{Ab}}^{+}(t)\right) d t}{\int_{1}^{\widehat{T}} I_{\mathrm{AB}}(t) H\left(I_{\mathrm{Ab}}^{-}(t)\right) d t}=\frac{\sum_{i=1}^{\widehat{T}} I_{\mathrm{Ab}}^{+}(t)}{\sum_{i=1}^{\widehat{T}} I_{\mathrm{Ab}}^{-}(t)},
$$

где $\widehat{\mathbb{T}}$ - количество часов расчетного периода, c; $I_{\mathrm{AБ}}(t)$ - зарядно-разрядная функция АБ; $I_{\mathrm{AБ}}^{+}(t)-$ функция заряда АБ; $I_{\mathrm{AБ}}^{-}(t)$ - функция разряда АБ. Функция $I_{\mathrm{AБ}}(t)$ и её составляющие $I_{\mathrm{Ab}}^{+}(t), I_{\mathrm{Ab}}^{-}(t)$ определяются относительно каждого временного интервала $(t) . H$ - функция Хэвисайда, где дискретная форма записи для процессов заряда и разряда АБ имеет следующий вид:

$$
\begin{aligned}
& H\left[I_{\mathrm{AБ}}^{-}(t)\right]=\left\{\begin{array}{ll}
0 ; & P_{S}(t) \geq 0 \\
1 ; & P_{S}(t)<0
\end{array}\right\}, \\
& H\left[I_{\mathrm{AБ}}^{+}(t)\right]=\left\{\begin{array}{ll}
0 ; & P_{S}(t) \leq 0 \\
1 ; & P_{S}(t)>0
\end{array}\right\} .
\end{aligned}
$$

Как отмечается в [23], большинство исследуемых АСЭС с ВИЭ имеют численное значение данного показателя от 1,02-1,30 о. е.

Коэффициент пропускной способности - выражается в виде суммарного годового разряда и приводится к установленной емкости АБ. Данный показатель необходимо использовать совместно с анализом количества часов при низкол заряде

$$
K_{\mathrm{AБ}}^{\Pi}=\frac{\int_{1}^{\widehat{T}} I_{\mathrm{Ab}}(t) H\left(I_{\mathrm{AБ}}^{-}(t)\right) d t}{Q_{\mathrm{Ab}}^{\max }}=\frac{\sum_{i=1}^{\widehat{T}} I_{\mathrm{Ab}}^{-}(t)}{Q_{\mathrm{AB}}^{\max }} .
$$

Данный показатель для АСЭС с ВИЭ, как правило, имеет значение от 10 до 100 о. е.

Коэффициент лаксилальной скорости разря$\partial a$ - демонстрирует максимальный ток разряда равным или превышающим $1 \%$ от $K_{\mathrm{AБ}}^{\mathrm{I}}$

$$
K_{\mathrm{AB}}^{P-\max }=\frac{0,01 \cdot K_{\mathrm{Ab}}^{\Pi} \cdot Q_{\mathrm{AB}}^{\max }}{\bar{a}_{\mathrm{AB}} \cdot \sum_{i=1}^{\mathbb{\pi}} t} .
$$

Данный показатель в первую очередь позволяет сделать вывод, насколько правильно выбрана суммарная установленная емкость АБ $Q_{\mathrm{AB}}^{\max }$ с учетом эксплуатационно-технических ограничений $\bar{k}_{\mathrm{AБ}}$. Также стоит отметить, что высокие значения разрядных токов существенно влияют на выбор суммарной установленной емкости АБ, где неспособность поддерживать высокие разрядные токи приводит к преждевременному старению и замене АБ.

Вреленной коэффициент полного заряда - характеризует среднее время между разрядом до минимально допустимого состояния заряда $Q_{\mathrm{Ab}}^{\text {min }}$ с последующим зарядом АБ до значений выше $90 \%$

$$
K_{\mathrm{AB}}^{B-\max } \frac{\int_{1}^{\widehat{T}} Q_{\mathrm{AB}}(t) H\left(Q_{\mathrm{AB}}^{\uparrow 90 \%}(t)\right) d t}{t_{\mathrm{AB}}^{\uparrow 90 \%}}=\frac{\sum_{i=1}^{\widehat{T}} Q_{\mathrm{AB}}^{\uparrow 90 \%}(t)}{t_{\mathrm{AB}}^{\uparrow 90 \%}} .
$$

Как правило, данный показатель составляет от 0,7 до 10 дней.

Коэффициент частичного заряда - представляет собой суммарную пропускную способность относительно состояния заряда АБ в моменты времени $(t)$

$$
K_{\mathrm{Ab}}^{Ц 3 ~}=\frac{\left(\begin{array}{l}
\left(k_{\mathrm{Ab}}^{85 \%-100 \%} \times 1\right)+\left(k_{\mathrm{Ab}}^{70 \%-85 \%} \times 2\right)+ \\
+\left(k_{\mathrm{Ab}}^{55 \%-70 \%} \times 3\right)+\left(k_{\mathrm{Ab}}^{40 \%-55 \%} \times 4\right)+ \\
+\left(k_{\mathrm{AB}}^{0 \%-40 \%} \times 5\right)
\end{array}\right)}{5},
$$

где $k_{\mathrm{AB}}{ }^{85 \%-100 \%}, k_{\mathrm{AB}}{ }^{75 \%-85 \%}, k_{\mathrm{AB}}{ }^{55 \%-70 \%}, k_{\mathrm{AB}}{ }^{40 \%-55 \%}, k_{\mathrm{AБ}}{ }^{0 \%-40 \%}$ являются коэффициентами, описывающими количество высвобождаемой энергии с соблюдением системы технических ограничений $\left(\bar{a}_{\mathrm{AБ}}\right)$ относительно рассматриваемого численного интервала состояния заряда в процентном соотношении. При этом универсальная форма записи данных коэффициентов имеет следующий вид:

$$
k_{\mathrm{Ab}}^{x-y}=\frac{\int_{1}^{\widehat{T}} I_{\mathrm{AБ}}(t) H\left(Q_{\mathrm{Ab}}^{x-y}(t)\right) H\left(I_{\mathrm{AБ}}^{-}(t)\right) d t}{\int_{1}^{\widehat{T}} I_{\mathrm{Ab}}(t) H\left(I_{\mathrm{Ab}}^{-}(t)\right) d t} \cdot 100,
$$

где $x, y$ - численное значение нижнего и верхнего порога соответственно, \% . 
Обычно данный коэффициент находится в диапазоне от 20 до $70 \%$

Вреленной коэффициент низкого заряда - оценивает среднегодовое количество часов, при которых состояние заряда АБ ниже $35 \%$

$$
K_{\mathrm{AB}}^{B-\min } \frac{\int_{1}^{\widehat{\pi}} Q_{\mathrm{AB}}(t) H\left(Q_{\mathrm{AB}}^{\downarrow 35 \%}(t)\right) d t}{\int_{1}^{\widehat{T}} d t}=\frac{\sum_{i=1}^{\widehat{T}} Q_{\mathrm{AB}}^{\downarrow 35 \%}(t)}{\widehat{\mathbb{T}}} .
$$

Данный показатель характеризует количество времени в году, при котором состояние заряда АБ $Q_{\mathrm{AБ}}(t)$ меньше $35 \%$, что приводит к сильной необратимой сульфации и кристаллизации.

В [23] отмечается, что не всегда имеется возможность фиксировать все из представленных показателей, что, несомненно, влияет на результат категоризации АБ.

После определения численных результатов коэффициентов происходит автоматический вывод оценок данного показателя по пятибалльной шкале. При этом оценки несут следующую смысловую нагрузку: 1 - очень низкая; 2 - низкая; 3 - средняя; 4 - высокая; 5 - очень высокая.

$$
\begin{aligned}
& 1 ;\left(K_{\mathrm{Ab}}^{3} \leq 102\right) \\
& \text { 2; }\left(K_{\mathrm{AB}}^{3}>102\right) \wedge\left(K_{\mathrm{AB}}^{3} \leq 108\right) \\
& \widehat{M}_{K_{\mathrm{AB}}^{3}}=\left\{3 ;\left(K_{\mathrm{Ab}}^{3}>108\right) \wedge\left(K_{\mathrm{Ab}}^{3} \leq 115\right)\right. \text {; } \\
& \text { 4; }\left(K_{\mathrm{AB}}^{3}>115\right) \wedge\left(K_{\mathrm{AB}}^{3} \leq 130\right) \\
& \text { 5; }\left(K_{\mathrm{Ab}}^{3}>130\right)
\end{aligned}
$$

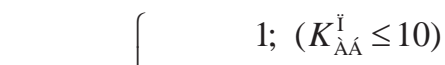

$$
\begin{aligned}
& \text { 2; }\left(K_{\mathrm{AB}}^{\Pi}>10\right) \wedge\left(K_{\mathrm{AB}}^{\Pi} \leq 40\right) \\
& \widehat{M}_{K_{\mathrm{AB}}^{\mathrm{I}}}= \begin{cases}2 ; & \left(K_{\mathrm{AB}}^{\Pi}>40\right) \wedge\left(K_{\mathrm{AB}}^{\Pi} \leq 70\right) ;\end{cases} \\
& \begin{array}{c}
4 ;\left(K_{\mathrm{AB}}^{\Pi}>70\right) \wedge\left(K_{\mathrm{AB}}^{\Pi} \leq 100\right) \\
5 ;\left(K_{\mathrm{AB}}^{\Pi}>100\right)
\end{array} \\
& 1 ;\left(K_{\mathrm{AB}}^{P-\max } \leq 0,1\right) \\
& \widehat{M}_{K_{\mathrm{AB}}^{P \max }}=\left\{\begin{array}{ll}
2 ; & \left(K_{\mathrm{Ab}}^{P-\max }>0,1\right) \wedge\left(K_{\mathrm{Ab}}^{P-\max } \leq 0,5\right) \\
3 ; & \left(K_{\mathrm{AB}}^{P-\max }>0,5\right) \wedge\left(K_{\mathrm{Ab}}^{P-\max } \leq 1,4\right) ;
\end{array}\right. \text {. } \\
& \text { 4; }\left(K_{\mathrm{AB}}^{P-\max }>1,4\right) \wedge\left(K_{\mathrm{AB}}^{P-\max } \leq 1,7\right) \\
& \text { 5; }\left(K_{\mathrm{Ab}}^{D-\max }>1,7\right)
\end{aligned}
$$

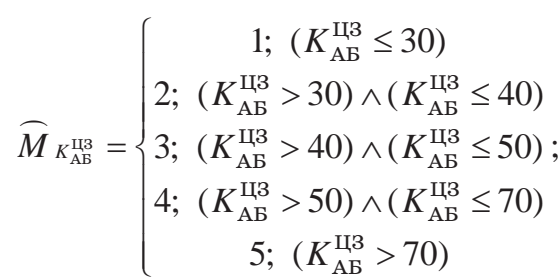

$$
\begin{aligned}
& \widehat{M}_{K_{\mathrm{AB}}^{B-\min }}=\left\{\begin{array}{c}
1 ;\left(K_{\mathrm{Ab}}^{B-\min } \leq 1\right) \\
2 ;\left(K_{\mathrm{Ab}}^{B-\min }>1\right) \wedge\left(K_{\mathrm{Ab}}^{B-\min } \leq 4,5\right) \\
3 ;\left(K_{\mathrm{AB}}^{B-\min }>4,5\right) \wedge\left(K_{\mathrm{Ab}}^{B-\min } \leq 15,5\right) ; \\
4 ;\left(K_{\mathrm{Ab}}^{B-\min }>15,5\right) \wedge\left(K_{\mathrm{Ab}}^{B-\min } \leq 25\right) \\
5 ;\left(K_{\mathrm{AB}}^{B-\min }>25\right)
\end{array}\right.
\end{aligned}
$$

$$
\widehat{M}_{K_{\mathrm{AB}}^{B-\max }}=\left\{\begin{array}{c}
1 ;\left(K_{\mathrm{Ab}}^{B-\max } \leq 0,7\right) \\
2 ; \quad\left(K_{\mathrm{AB}}^{B-\max }>0,7\right) \wedge\left(K_{\mathrm{AB}}^{B-\max } \leq 1,2\right) \\
3 ;\left(K_{\mathrm{AB}}^{B-\max }>1,2\right) \wedge\left(K_{\mathrm{AB}}^{B-\max } \leq 2,5\right) \\
4 ;\left(K_{\mathrm{AB}}^{B-\max }>2,5\right) \wedge\left(K_{\mathrm{AB}}^{B-\max } \leq 8\right) \\
5 ;\left(K_{\mathrm{AB}}^{B-\max }>8\right)
\end{array}\right.
$$

При этом анализ полученных показателей позволяет в целом определить необходимые условия для эксплуатации АБ и снизить негативные воздействия на активную массу. Более детально с процессом категоризаиии можно ознакомиться в работах $[23,29,30]$.

\section{Интеграция процесса категоризации в задачи}

оптимизации аккумуляторных батарей в АСЭС с ВИЭ

Процесс категоризаиии АБ является сложным и требует большого количества первоначальной информации. В то же время хорошая проработка химической составляющей влияния режимных параметров на процессы деградации активной массы АБ показывает состоятельность предлагаемой методики.

Важно отметить, что зачастую детализированной первоначальной информации нет в наличии. Кроме того, процесс категоризации напрямую зависит от временных интервалов $(t)$, при которых генерация ВИЭ имеет стохастический характер, что, несомненно, влияет на знакопеременную функцию мощности $P_{S}(t)$. При этом немаловажное значение имеет нагрузочная характеристика потребителя, которая также зависит от времени года, месяца, дня и часа расчетного периода. Поэтому, согласно [23], можно сделать вывод, что в целом численные значения процесса категоризации могут варьироваться в зависимости от конфигурации сети, логических условий управления, нагрузочной характеристики потребителя, состава оборудования, природно-климатических показателей и т. д.

В то же время автор статьи утверждает, что при наличии детализированной модели АСЭС с ВИЭ и АБ с интеграцией реальных метеорологических рядов и применении хронологического метода расчета системы с фиксацией необходимых режимных параметров относительно временного интервала $(t)$ возможно использование процесса категоризации при решении задачи оптимизации АБ в АСЭС с ВИЭ.

\section{Содержательное описание предлагаемого подхода}

Предлагается производить моделирование АСЭС с ВИЭ и АБ на протяжении всего количества лет метеорологических наблюдений с дискретным шагом один час. При этом все метеорологические параметры относительно каждого временного интервала $(t)$ принимаются равными численным значениям, замеренным на соответствующем временном отрезке. Данные массивы автоматически обрабатываются и приводятся к одному временному интервалу в зависимости от первоначального метеорологического кода (FM 12 Synop или METAR), 
описывающего массив природно-климатических показателей.

Обработка метеорологических рядов производится автоматически с применением методики и реализующей её ПВК «Локальный анализ параметров окружающей среды и солнечной радиации», в рамках которого также моделируются актинометрические показатели, такие как прямая, рассеянная и суммарная солнечная радиация с учетом фактической облачности на рассматриваемой территории. При определении актинометрических показателей используется тандел математических моделей Iqbal и Kasten-Czeplak [33, 34].

После того как сформирован итоговый много летний массив паралетров окружающей среды, включающий актинометрические, ветроэнергетические и другие природно-климатические показатели, производится сквозное моделирование АСЭС с шагом один час на всю глубину метеорологических наблюдений. При этом относительно каждого временного интервала $(t)$ определяется уровень генерации ВЭУ, ФЭП, ДГ в зависимости от условий управления АСЭС и других режимных параметров. Также определяются значения знакопереленной функиии мощности $P_{S}(t)$ системы с учетом нагрузочной характеристики потребителя $P_{H}(t)$ и потерь электроэнергии $\Delta P_{\Sigma}(t)$ относительно каждого временного интервала с последующим разделением на положительную и отрицательную области. В то же время эксплуатационно-технические ограничения $\bar{a}_{\mathrm{AБ}}$ по величинам заряда и разряда АБ принимаются равными общепринятым значениям.

На конец расчетного периода относительно знакопеременной функиии мощности $P_{S}(t)$, состояния заряда АБ $Q_{\mathrm{AБ}}(t)$ и фактических величин тока заряда $I_{\mathrm{AB}}^{+}(t)$ и разряда $I_{\mathrm{AБ}}^{-}(t)$ определяются описанные ранее коэффициенты $K_{\mathrm{Ab}}^{3}, K_{\mathrm{Ab}}^{\Pi}, K_{\mathrm{Ab}}^{p-\max }, K_{\mathrm{Ab}}^{B-\max }$, $K_{\mathrm{Ab}}^{ц 3}, K_{\mathrm{AБ}}^{B \text {-min }}$, необходимые для категоризации АБ. Численные результаты полученных коэффициентов сравниваются с референтными значениями, и производится расчет оценок $\widehat{M}_{K_{A \mathrm{~B}}^{3}}, \widehat{M}_{K_{A \mathrm{~A}}^{\mathrm{I}}}, \widehat{M}_{K_{\mathrm{AB}}^{\mathrm{p} \text {-max }}}, \widehat{M}_{K_{\mathrm{AB}}^{B-\max }}$, $\widehat{M}_{K_{s}^{\amalg 3}}, \widehat{M}_{K_{s}^{B-\text { min }}}$, где на их основании формируется итоговый результат категоризации АБ. По итогам автоматического анализа и построения графического изображения полученных оценок определяется номер категории АБ. На рис. 4 показаны диаграммы шести категорий АБ [23].

При этом на основании полученных оценок и определения номера категории формируется описание и степень возникновения основных процессов деградации активной массы АБ, что автоматически позволяет сформировать потенциально возможные варианты компоновки электрохимических накопителей энергии, в которых данные негативные процессы сведены к минилулу. Такой подход позволяет заранее отсечь те типы АБ, которые при данных условиях эксплуатации сильно подвержены негативным воздействиям деградации.

В табл. 1 показаны основные процессы деградации активной массы АБ и степень их возникновения в зависимости от полученной категории [23].
Таблииа 1. Процессы деградации и степень их возникновения для различных категорий

Table 1. Degradation and its probability for different categories

\begin{tabular}{|l|c|c|c|c|c|c|}
\hline \multirow{2}{*}{$\begin{array}{l}\text { Процесс деградации } \\
\text { Degradation }\end{array}$} & \multicolumn{5}{|c|}{$\begin{array}{c}\text { Номер категории } \\
\text { Category number }\end{array}$} \\
\cline { 2 - 8 } & 1 & 2 & 3 & 4 & 5 & 6 \\
\hline $\begin{array}{l}\text { Kopрозия положительной пластины } \\
\text { Corrosion of the positive grid }\end{array}$ & 1 & 4 & 5 & 4 & 3 & 2 \\
\hline $\begin{array}{l}\text { Tяжелая/необратимая сульфация } \\
\text { Нard/irreversible sulphation }\end{array}$ & 5 & 1 & 1 & 3 & 3 & 4 \\
\hline $\begin{array}{l}\text { Bыпадение активной массы из пластин } \\
\text { Shedding }\end{array}$ & 4 & 1 & 4 & 5 & 3 & 3 \\
\hline $\begin{array}{l}\text { Потеря жидкости } \\
\text { Water loss }\end{array}$ & 1 & 4 & 5 & 4 & 3 & 2 \\
\hline $\begin{array}{l}\text { Paзрушение активной массы } \\
\text { Destruction of the асtive таss }\end{array}$ & 5 & 1 & 2 & 3 & 3 & 3 \\
\hline $\begin{array}{l}\text { Стратификация электролита } \\
\text { Electrolyte stratification }\end{array}$ & 5 & 1 & 1 & 4 & 3 & 4 \\
\hline
\end{tabular}

1 - очень низкая/very low; 2 - низкая/low; 3 - средняя/medium; 4 высокая/high; 5 - очень высокая/vеry high.

Таким образом, совместное использование детализированной модели АСЭС с ВИЭ и АБ, дополненной многолетними метеорологическими рядами с определением основных режимных параметров с соблюдением ряда эксплуатационно-технических ограничений как по отдельным элементам, так и всей системы в целом с последующей категоризацией АБ, позволяет выделить максимально пригодные для данных условий типы (технологии) электрохимических накопителей энергии.

\section{Программно-вычислительный комплекс}

Представленный в рамках данной статьи подход реализован в ПВК «Выбор электрохилических накопителей энергии». ПВК состоит из следующих укрупненных информационных блоков, в которых выполняются следующие действия: грynna 1 - сбор первоначальной (режимной) информации относительно каждого временного интервала $(t)$, , зн $a$ копеременной функции мощности $P_{S}(t)$, состояния заряда АБ $Q_{\mathrm{AБ}}(t)$ и величин тока заряда $I_{\mathrm{AБ}}^{+}(t)$ и разряда $I_{\mathrm{Ab}}^{-}(t)$; группа 2 - обработка полученной информации (формирование структуры обрабатываемого массива), передача подготовленного массива для дальнейших операций; группа 3 - определение коэффициентов $K_{\mathrm{AB}}^{3}, K_{\mathrm{AB}}^{\mathrm{II}}, K_{\mathrm{AB}}^{p \text {-max }}, K_{\mathrm{Ab}}^{B \text {-max }}, K_{\mathrm{AB}}^{\amalg 3}, K_{\mathrm{AB}}^{B-\min }$ с последующим расчетом оценок $\widehat{M}_{K_{A B}^{3}}, \widehat{M}_{K_{A B}^{\Pi}}, \widehat{M}_{K_{A \mathrm{~B}}^{p-\max }}, \widehat{M}_{K_{A \mathrm{~B}}^{B-\max }}, M_{K_{A \mathrm{~B}}^{\amalg}}^{\text {, }}$ $\widehat{M}_{K A Б B-\min }$ и определением номера категории АБ; груnпа 4 - выполняет действия визуализации и представления полученных результатов процесса категоризации АБ; группа 5 - является базой данных матричного вида, в которой хранится информация о различных видах АБ.

Работу ПВК можно описать следующей укрупненной блок-схемой (рис. 5).

\section{Объекты исследования}

В качестве объектов исследования были выбраны три АСЭС, условно расположенные в различных природно-климатических зонах. При этом анализ природно-климатических показателей выполнялся с использованием метеорологических рядов, 
Категория 1

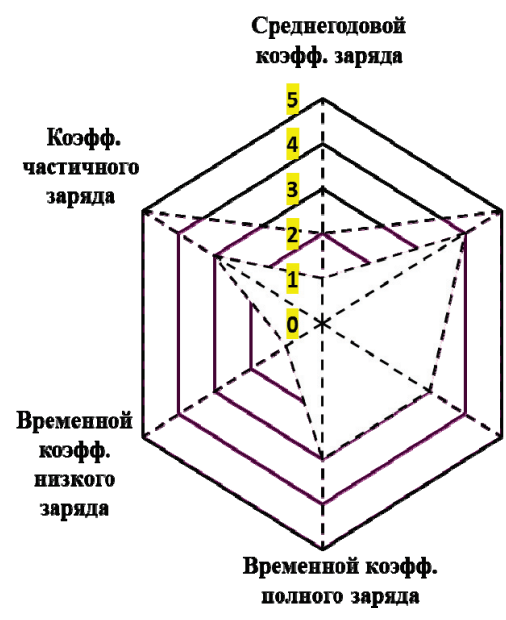

Категория 3

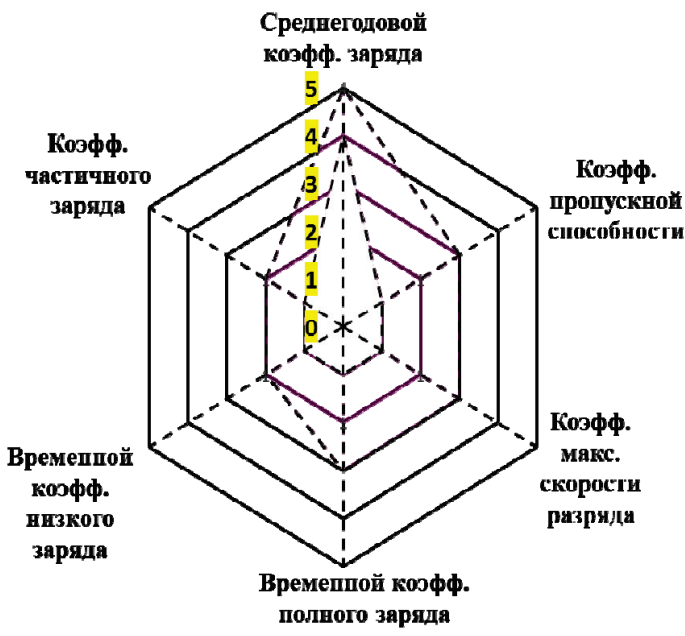

Категория 5

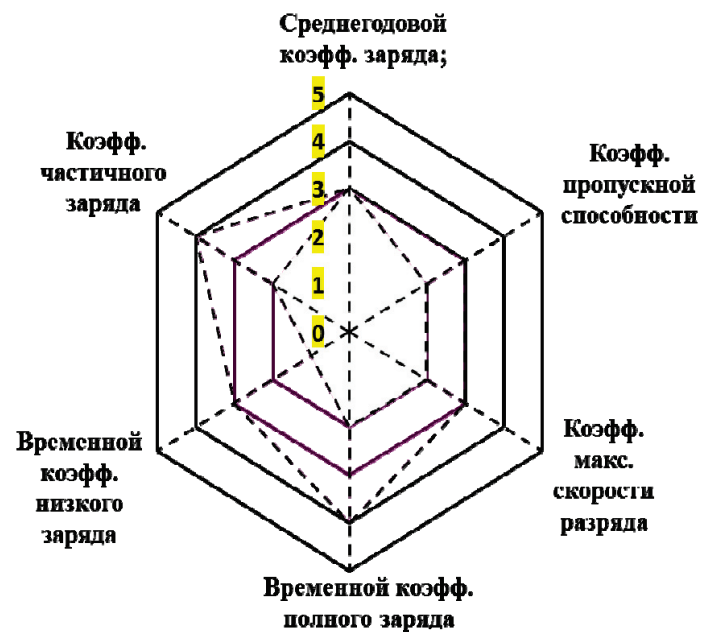

Категория 2

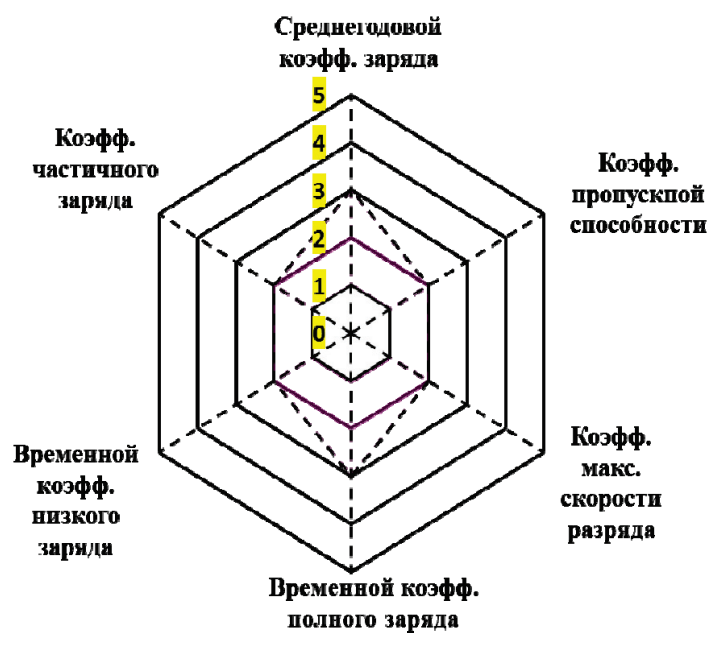

Категория 4

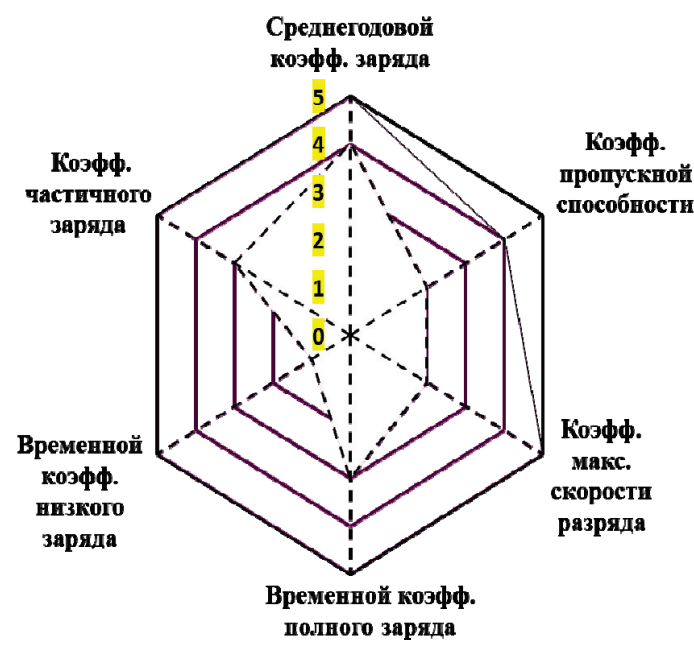

Категория 6

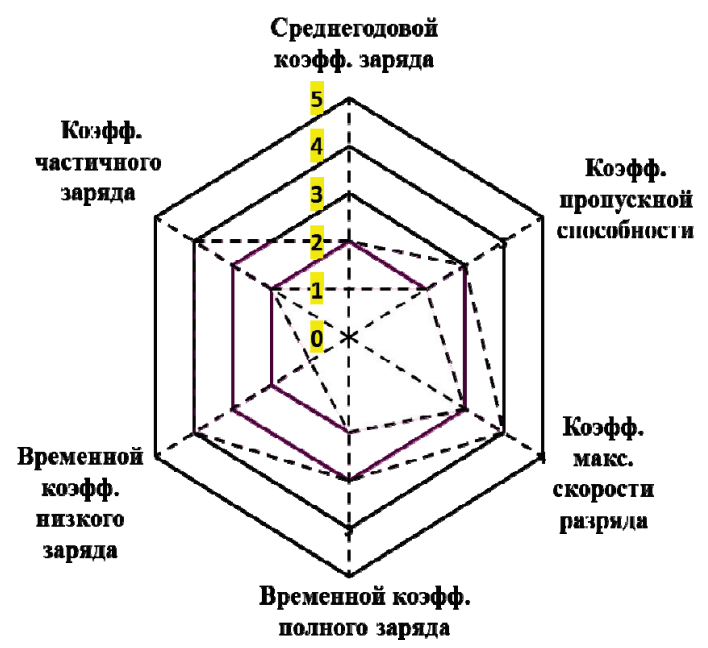

*Розовым ияветом показана рабочая область категории/Operaing region of the gategry is shown in pink

Pис. 4. Диаграммы шести категорий АБ в зависимости от условий эксплуатации

Fig. 4. Diagrams of six categories of storage batteries, depending on the operating conditions 


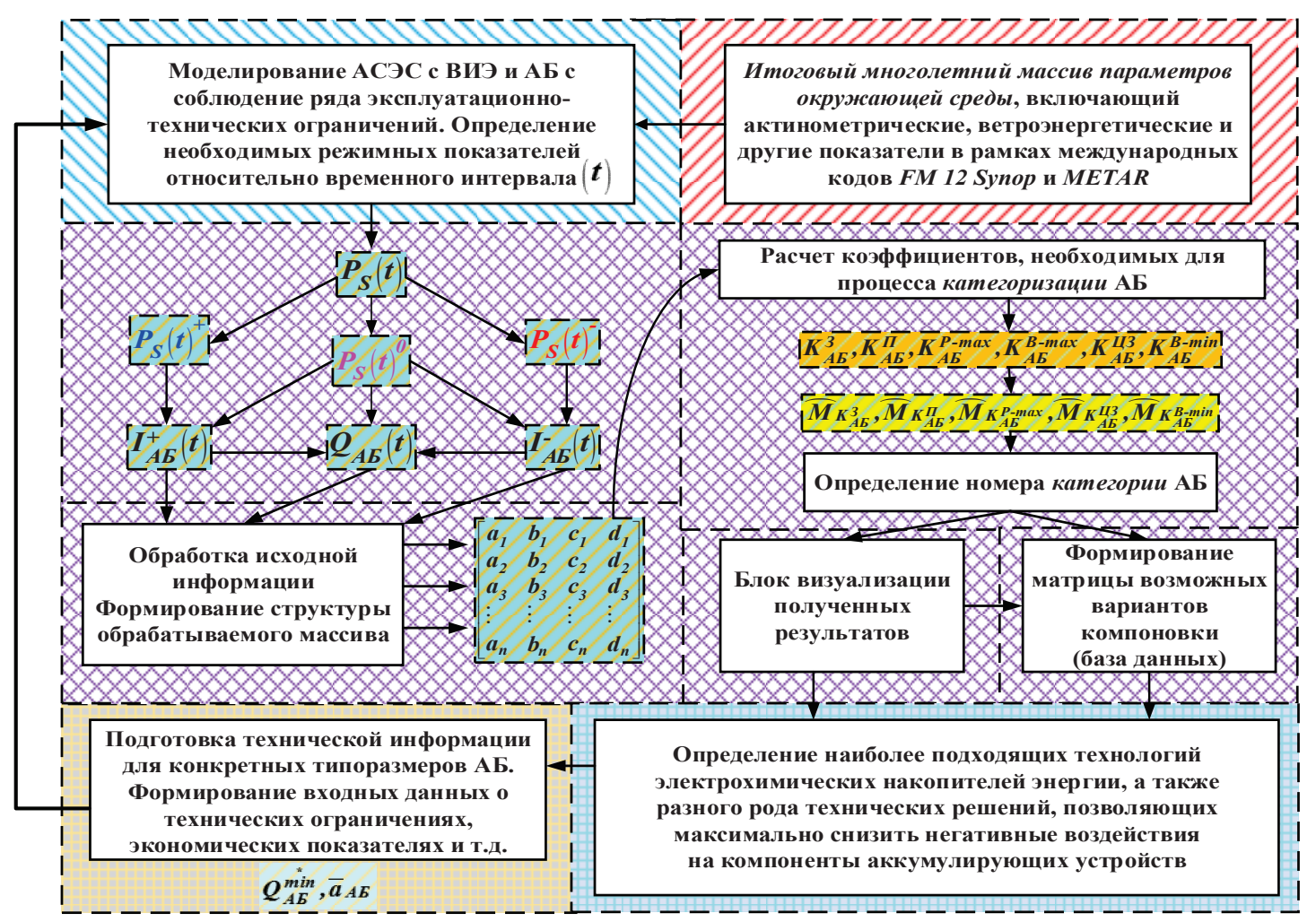

Рис.5. Блок-схема, укрупненно описывающал работу ПВК «Выбор электрохилических накопителей энергии»

Fig. 5. Block-diagram describing basic steps of operation of the software «Selection of electrochemical energy storage»

зафиксированных на ближайшей от рассматриваемой системы метеостанции. Относительно каждого временного интервала $(t)$ определяются актинометрические, ветроэнергетические и многие другие метеопараметры, необходимые для моделирования.

Нагрузочная характеристика потребителя для всех трех вариантов принималась одинаковой, с максимальным и минимальным уровнем в зимний и летний периоды соответственно. Данное условие позволяет продемонстрировать влияние состава и установленной мощности генерирующего сетевого оборудования с учетом реальной природно-климатической обстановки на рассматриваемой территории на процесс категоризации электрохимических накопителей энергии.

На рис. 6 показана нагрузочная характеристика, используемая при моделировании АСЭС.

Согласно первоначальным условиям, принимается, что в рассматриваемых АСЭС уже имеется исходный состав генерирующего оборудования (ВЭУ, ФЭП, ДГ), а также элементы распределительной сети. Соответственно, следующим этапом является выбор необходимой емкости АБ с учетом фактических условий эксплуатации, влияющих на процесс категоризации АБ. Данное условие можно представить как многоитерационный процесс оптимизации состава оборудования с применением метода Гаусса-Зейделя, при котором на первых итерациях были определены и зафиксированы оптимальные установленные мощности основного состава оборудования с последующим на- хождением оптимальной емкости АБ с учетом полученной категории. Шаг установленной емкости АБ равен 1000 А·ч, что при схеме соединения под напряжение $48 \mathrm{~B}$ составляет 48 кВт·ч.

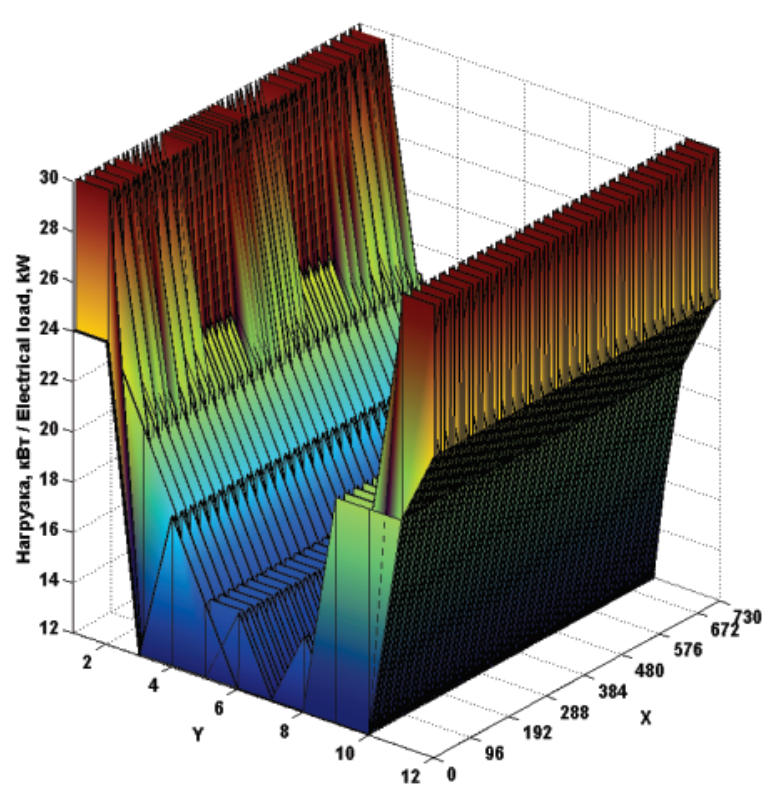

$X$ - номер часа/Number of hour,

$Y$ - номер месяиа/Number of month

Puс. 6. Нагрузочная характеристика, используемая при моделировании АСЭС

Fig. 6. Electrical load used in modelling 
В работе использовались природно-климатические показатели, полученные для н.п. Ном, Коди$а \kappa$, Адак, расположенных в американском штате Аляска. Природно-климатические показатели были определены автором в предыдущих исследованиях [34].

\section{Численные результаты}

- АСЭС № 1 - Ном. Согласно принятым допущениям в АСЭС имеется следующий первоначальный состав энергетического оборудования: ВЭУ - $4 \times 30(120)$ кВт; ДГ - $1 \times 75$. Согласно рис. 5, на первом этапе задается условная АБ с общеизвестными ограничениями $\bar{a}_{\mathrm{AБ}}$.

На рис. 7, 8 показаны характеристики генерации ВЭУ и ДГ при различных установленных емкостях АБ для АСЭС № 1 - Ном.

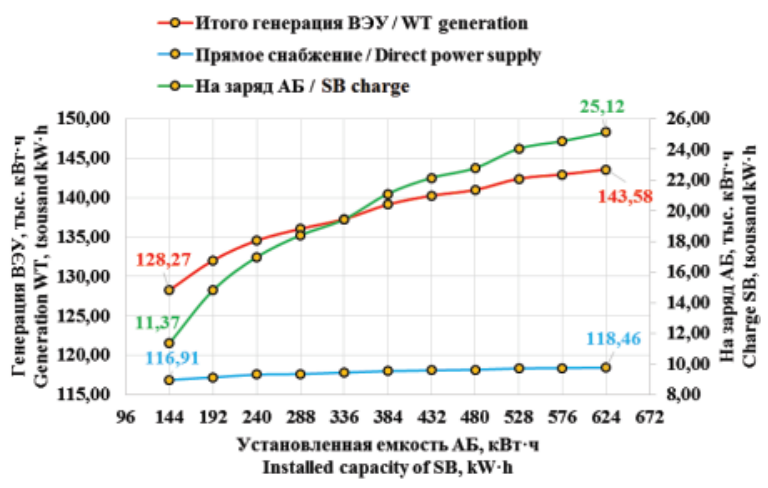

Pис. 7. Характеристики генерации ВЭУ при различных установленных емкостлх $А Б$

Fig. 7. Characteristics of WT generation $(4 \times 30 \mathrm{~kW})$ at various installed capacity of $S B$

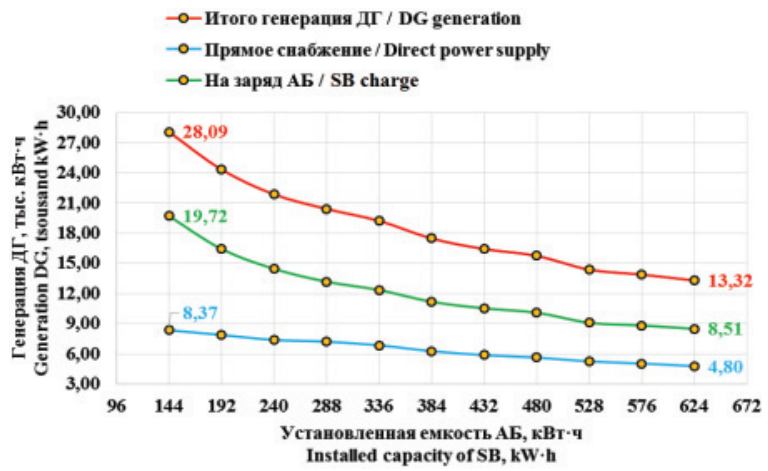

Pис. 8. Характеристики генерации ДГ при различных установленных емкостах $А Б$

Fig. 8. Characteristics of $D G$ generation at various installed capacity of $S B$

Как видно из полученных результатов, увеличение установленной емкости АБ позволяет для АСЭС № 1 - Нол увеличить генерацию ВЭУ, повысить маневренность АСЭС, при этом существенно сократить число часов работы и соответственно генерацию ДГ.

При этом анализ оценок $\widehat{M}_{K_{\mathrm{AB}}^{3}}, \widehat{M}_{K_{\mathrm{AB}}^{\mathrm{I}}}, \widehat{M}_{K_{\mathrm{AB}}^{P-\max }}, \widehat{M}_{K_{\mathrm{AB}}^{B-\max }}$, $\widehat{M}_{K_{\mathrm{AB}}^{\amalg 3}}, \widehat{M}_{K_{A \mathrm{~B}}^{-\mathrm{min}}}$ относительно каждой итерации расчета имеет следующую картину (табл. 2).
Таблица 2. Численные значения оценок коэффициентов, характеризующих процесс категоризации АБ относительно установленной емкости $А Б$

Table 2. Numerical values of estimates coefficients characterizing $S B$ categorization relative to the installed capacity of $S B$

\begin{tabular}{|c|c|c|c|c|c|c|}
\hline \multirow{2}{*}{$\begin{array}{l}\text { Уст. емкость АБ, кВт॰ч } \\
\text { Ins. capacity of } \mathrm{SB}, \mathrm{kW} \cdot \mathrm{h}\end{array}$} & \multicolumn{6}{|c|}{ Оценки/Estimates } \\
\hline & $\widehat{M}_{K_{A B}^{3}}$ & $\widehat{M}_{K_{\mathrm{AB}}^{\mathrm{I}}}$ & $\widehat{M}_{K_{A B}^{p-\max }}$ & $\widehat{M}_{K_{A \mathrm{~B}}^{B \max }}$ & $\widehat{M}_{K_{A B}^{B-\min }}$ & $\widehat{M}_{K_{A \mathrm{~B}}^{\text {म3 }}}$ \\
\hline 144 & 2 & 5 & 5 & 3 & 2 & 5 \\
\hline 192 & 2 & 5 & 4 & 3 & 2 & 4 \\
\hline 240 & 2 & 5 & 3 & 3 & 2 & 4 \\
\hline 288 & 2 & 5 & 3 & 3 & 1 & 4 \\
\hline 336 & 1 & 4 & 3 & 3 & 1 & 4 \\
\hline 384 & 1 & 4 & 3 & 3 & 1 & 3 \\
\hline 432 & 1 & 4 & 3 & 3 & 1 & 3 \\
\hline 480 & 1 & 3 & 3 & 3 & 1 & 3 \\
\hline 528 & 1 & 3 & 3 & 3 & 1 & 3 \\
\hline 576 & 1 & 3 & 3 & 3 & 1 & 3 \\
\hline 624 & 1 & 3 & 3 & 3 & 1 & 3 \\
\hline
\end{tabular}

В целом полученные значения оценок показывают, что изменение установленной емкости АБ существенно влияет на режимные эксплуатационные параметры рассматриваемой системы. При этом увеличение установленной емкости АБ снижает численные значения полученных оценок, что существенно сказывается на процессе идентификации вектора оценок $\widehat{M}_{K_{A B}^{3}}, \widehat{M}_{K_{A \mathrm{~B}}^{\Pi}}, \widehat{M}_{K_{\mathrm{AB}}^{p-\max }}, \widehat{M}_{K_{\mathrm{AB}}^{B-\max }}, \widehat{M}_{K_{A \mathrm{~B}}^{\amalg}}^{\text {, }}$, $\widehat{M}_{K_{A B}^{B-\text { min }}}$ с последующим выводом о номере категории.

В табл. 3 показано соответствие полученных численных значений оценок относительно каждой итерации расчета.

Таблица 3. Степень совпадения оценочных коэффициентов с референтныли значенияли относительно установленной емкости АБ и номера категории

Table 3. Degree of coincidence of the estimated coefficients with reference values relative to the installed capacity of $S B$ and the category number

\begin{tabular}{|c|c|c|c|c|c|c|}
\hline \multirow{3}{*}{$\begin{array}{l}\text { Уст. емкость АБ, кВт॰ч } \\
\text { Ins. capacity of SB, kW·h }\end{array}$} & \multicolumn{6}{|c|}{ Номер категории/Category number } \\
\hline & 1 & 2 & 3 & 4 & 5 & 6 \\
\hline & \multicolumn{6}{|c|}{$\%$} \\
\hline 144 & \multirow{7}{*}{100} & \multirow{11}{*}{50} & \multirow{2}{*}{33} & \multirow{4}{*}{67} & 33 & 50 \\
\hline 192 & & & & & 50 & \multirow{6}{*}{83} \\
\hline 240 & & & \multirow{5}{*}{50} & & \multirow{5}{*}{67} & \\
\hline 288 & & & & & & \\
\hline 336 & & & & & & \\
\hline 384 & & & & & & \\
\hline 432 & & & & & & \\
\hline 480 & \multirow{4}{*}{83} & & \multirow{4}{*}{67} & 83 & \multirow{4}{*}{83} & \multirow{4}{*}{100} \\
\hline 528 & & & & & & \\
\hline 576 & & & & & & \\
\hline 624 & & & & & & \\
\hline
\end{tabular}

Результаты категоризации АБ для АСЭС № 1 Нол позволяют сделать следующие выводы:

- при установленной емкости АБ от 144 до 432 кВт•ч режимы работы аккумулятора соответствуют 1 категории;

- начиная с $480 \kappa \mathrm{KT}^{*} ч$ режимы работы АБ соответствуют 6 категории;

- АCЭС № 2 - Кодиак. 
С учетом озвученных допущений было принято, что первоначальный состав генерирующего оборудования составляет 100 кВт ФЭП и ДГ 1?75. При этом моделирование рассматриваемой системы для метеорологических условий $А С Э С$ № 2 - Кодиак показывает следующий характер (рис. 9, 10).

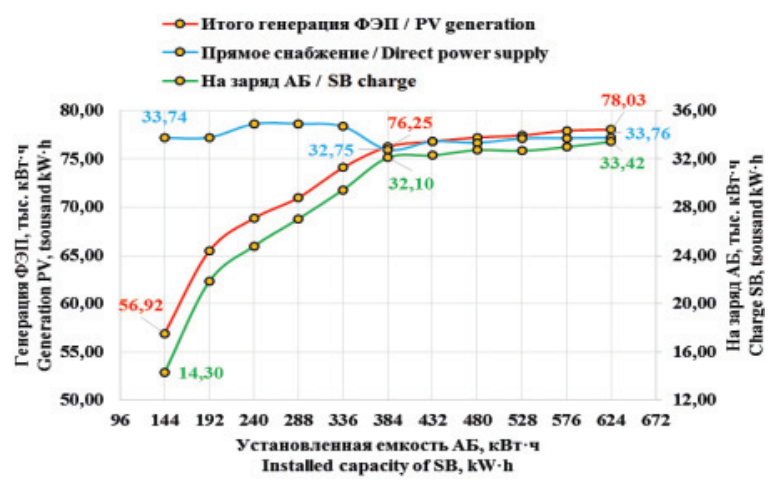

Pис.9. Характеристики генерации ФЭП при различных установленных емкостах АБ

Fig. 9. Characteristics of $P V$ generation $(100 \mathrm{~kW})$ at various installed capacity of $S B$

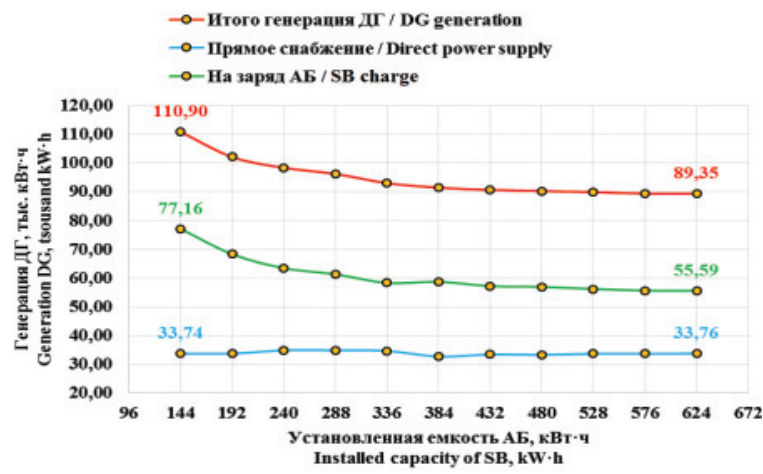

Рис. 10. Характеристики генерации ДГ при различных установленных емкостах АБ

Fig. 10. Characteristics of DG generation at various installed capacity of $S B$

В табл. 4 показаны полученные оценки относительно различных величин установленных емкостей АБ.

В табл. 5 показано соответствие полученных численных значений оценок относительно каждой итерации расчета.

Полученные результаты показывают, что ис-

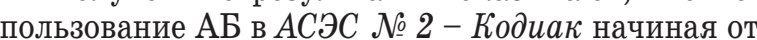
144 до 624 кВт॰ч имеют $100 \%$ совпадение с 1 кате горией.

- АCЭС № $3-А \partial а \kappa$.

Согласно исходному сценарию, имеется следующий состав генерирующего оборудования:

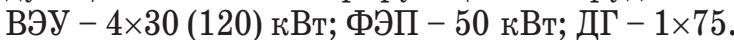

На рис. 11-13 показаны характеристики генерации ВЭУ, ФЭП и ДГ при различной установленной емкости АБ.

В табл. 6 показаны полученные оценки относительно различных величин установленных емкостей АБ.
Таблииа 4. Численные значения оценок коэффиииентов, характеризующих процесс категоризации АБ относитель но установленной емкости АБ

Table 4. Numerical values of estimates coefficients characterizing $S B$ categorization relative to the installed capacity of $S B$

\begin{tabular}{|c|c|c|c|c|c|c|}
\hline \multirow{2}{*}{$\begin{array}{l}\text { Уст. емкость АБ, кВт॰ч } \\
\text { Ins. capacity of SB, kW·h }\end{array}$} & \multicolumn{6}{|c|}{ Оценки/Estimates } \\
\hline & $M_{K_{\mathrm{AB}}^{3}}$ & $\widehat{M}_{K_{\mathrm{AB}}^{\mathrm{I}}}$ & $\widehat{M}_{K_{A B}^{P_{\text {max }}}}$ & $M_{r_{A B}^{B} \max }$ & $\widehat{M}_{K_{\mathrm{AB}}^{B-\min }}$ & $M_{K_{A B}^{\text {प3 }}}$ \\
\hline 144 & 1 & 5 & 5 & 4 & 3 & 4 \\
\hline 192 & 1 & 5 & 5 & 4 & 3 & 5 \\
\hline 240 & 1 & 5 & 5 & 4 & 2 & 5 \\
\hline 288 & 1 & 5 & 5 & 4 & 2 & 4 \\
\hline 336 & 1 & 5 & 5 & 4 & 2 & 4 \\
\hline 384 & 1 & 5 & 5 & 4 & 2 & 4 \\
\hline 432 & 1 & 5 & 5 & 4 & 2 & 4 \\
\hline 480 & 1 & 5 & 5 & 4 & 2 & 4 \\
\hline 528 & 1 & 5 & 4 & 4 & 2 & 4 \\
\hline 576 & 1 & 5 & 4 & 4 & 2 & 4 \\
\hline 624 & 1 & 5 & 4 & 4 & 2 & 4 \\
\hline
\end{tabular}

Таблица 5. Степень совпадения оценочных коэффициентов с ре ферентныли значенияли относительно устано вленной елкости АБ и номера категории

Table 5. Degree of coincidence of the estimated coefficients with reference values relative to the installed capacity of $S B$ and the category number

\begin{tabular}{|c|c|c|c|c|c|c|}
\hline \multirow{3}{*}{$\begin{array}{l}\text { Уст. емкость АБ, кВт•ч } \\
\text { Ins. capacity of } \mathrm{SB}, \mathrm{kW} \cdot \mathrm{h}\end{array}$} & \multicolumn{6}{|c|}{ Номер категории/Category number } \\
\hline & 1 & 2 & 3 & 4 & 5 & 6 \\
\hline & \multicolumn{6}{|c|}{$\%$} \\
\hline 144 & \multirow{11}{*}{100} & \multirow{2}{*}{17} & \multirow{2}{*}{0} & & 50 & 50 \\
\hline 192 & & & & & \multirow{2}{*}{33} & \multirow{2}{*}{33} \\
\hline 240 & & \multirow{9}{*}{33} & \multirow{9}{*}{17} & & & \\
\hline 288 & & & & & \multirow{8}{*}{50} & \multirow{5}{*}{50} \\
\hline 336 & & & & & & \\
\hline 384 & & & & 67 & & \\
\hline 432 & & & & & & \\
\hline 480 & & & & & & \\
\hline 528 & & & & & & \multirow{3}{*}{67} \\
\hline 576 & & & & & & \\
\hline 624 & & & & & & \\
\hline
\end{tabular}

Таблица 6. Численные значения оценок коэффициентов, харак теризуюших проиесс категоризации АБ относительно установленной емкости АБ

Table 6. Numerical values of coefficients estimates characterizing the process of categorization of $S B$ relative to the installed capacity of $S B$

\begin{tabular}{|c|c|c|c|c|c|c|}
\hline \multirow{2}{*}{$\begin{array}{l}\text { Уст. емкость АБ, кВт॰ч } \\
\text { Ins. capacity of SB, kW·h }\end{array}$} & \multicolumn{6}{|c|}{ Оценки/Estimates } \\
\hline & $M_{K_{\mathrm{AB}}^{3}}$ & $M_{K_{A 5}^{\mathrm{H}}}$ & $M_{K_{\mathrm{AB}}^{p-\max }}$ & $M_{K_{\mathrm{AB}}^{B-\max }}$ & $M_{K_{A \mathrm{~B}}^{\text {B-in }}}$ & $M_{K_{A 5}^{\text {प3 }}}$ \\
\hline 144 & 2 & 5 & 5 & 3 & 2 & 4 \\
\hline 192 & 2 & 5 & 5 & 3 & 2 & 4 \\
\hline 240 & 2 & 5 & 4 & 3 & 2 & 4 \\
\hline 288 & 2 & 5 & 3 & 3 & 1 & 4 \\
\hline 336 & 2 & 5 & 3 & 3 & 1 & 4 \\
\hline 384 & 1 & 4 & 3 & 3 & 1 & 4 \\
\hline 432 & 1 & 4 & 3 & 3 & 1 & 4 \\
\hline 480 & 1 & 4 & 3 & 3 & 1 & 4 \\
\hline 528 & 1 & 3 & 3 & 3 & 1 & 3 \\
\hline 576 & 1 & 3 & 3 & 3 & 1 & 3 \\
\hline 624 & 1 & 3 & 3 & 3 & 1 & 3 \\
\hline
\end{tabular}

В табл. 7 показано соответствие полученных численных значений оценок относительно каждой итерации расчета. 
Таблица 7. Степень совпадения оценочных коэффициентов с ре ферентными значениями относительно устано вленной емкости АБ и номера категории

Table 7. Degree of coincidence of the estimated coefficients with reference values relative to the installed capacity of the $S B$ and the category number

\begin{tabular}{|c|c|c|c|c|c|c|}
\hline \multirow{3}{*}{$\begin{array}{l}\text { Уст. емкость АБ, кВт॰ч } \\
\text { Ins. capacity of } \mathrm{SB}, \mathrm{kW} \cdot \mathrm{h}\end{array}$} & \multicolumn{6}{|c|}{ Номер категории/Category number } \\
\hline & 1 & 2 & 3 & 4 & 5 & 6 \\
\hline & \multicolumn{6}{|c|}{$\%$} \\
\hline 144 & \multirow{8}{*}{100} & \multirow{11}{*}{50} & \multirow{3}{*}{33} & \multirow{5}{*}{67} & \multirow{3}{*}{50} & \multirow{2}{*}{67} \\
\hline 192 & & & & & & \\
\hline 240 & & & & & & \multirow{6}{*}{83} \\
\hline 288 & & & \multirow{5}{*}{50} & & \multirow{5}{*}{67} & \\
\hline 336 & & & & & & \\
\hline 384 & & & & \multirow{6}{*}{83} & & \\
\hline 432 & & & & & & \\
\hline 480 & & & & & & \\
\hline 528 & \multirow{3}{*}{83} & & \multirow{3}{*}{67} & & \multirow{3}{*}{83} & \multirow{3}{*}{100} \\
\hline 576 & & & & & & \\
\hline 624 & & & & & & \\
\hline
\end{tabular}

Результаты категоризащии АБ для АСЭС № 3 Адак позволяют сделать следующие выводы:

- при установленной емкости АБ от 144 до 480 кВт॰ч режимы работы аккумулятора соответствуют 1 категории;

- начиная с $528 \mathrm{\kappa Bт} \cdot ч$ режимы работы АБ соответствуют 6 категории.

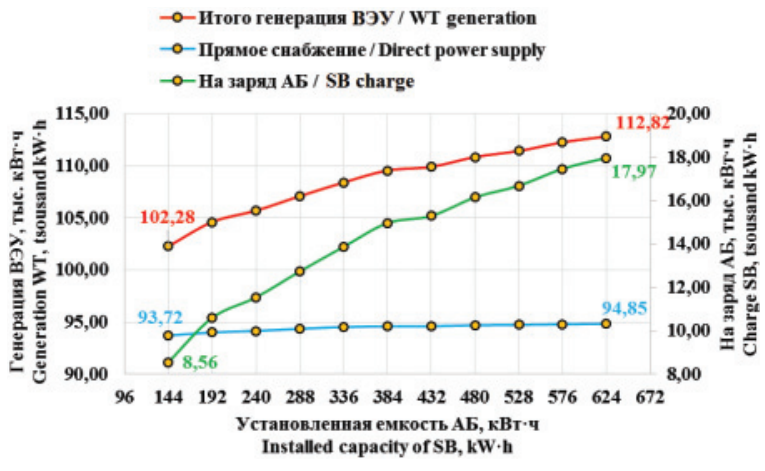

Рис. 11. Характеристики генерации ВЭУ при различных установленных емкостях АБ

Fig. 11. Characteristics of WT generation $(4 \times 30 \mathrm{~kW})$ at various installed capacity of $S B$

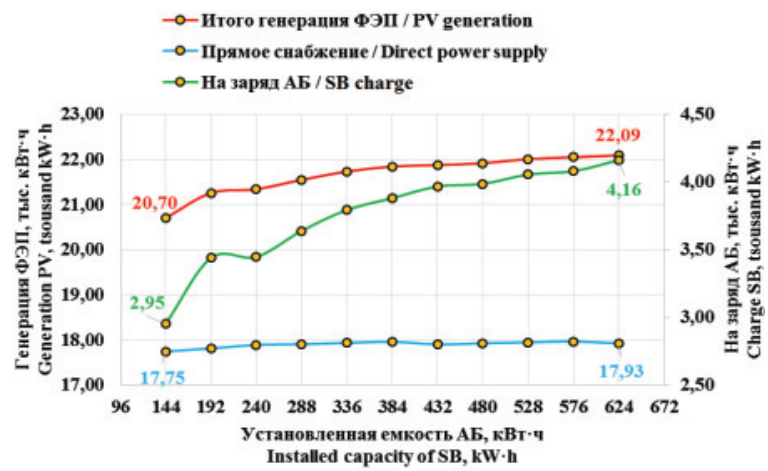

Рис. 12. Характеристики генерации ФЭП при различных уста новленных елкостях $А Б$

Fig. 12. Characteristics of $P V$ generation $(50 \mathrm{~kW})$ at various instal led capacity of $S B$

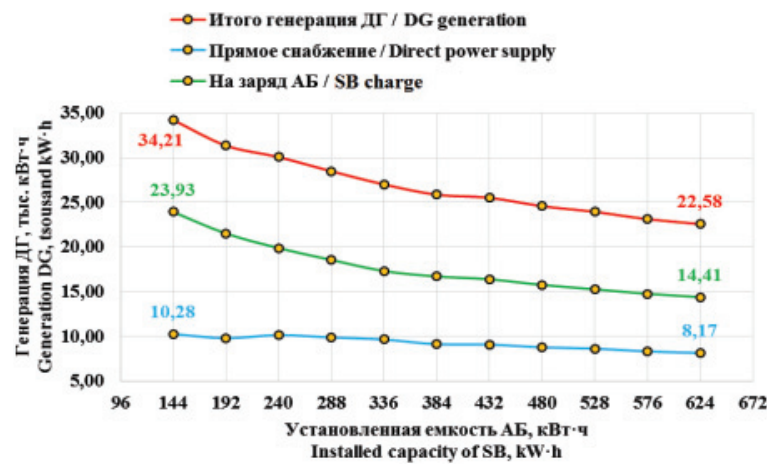

Рис. 13. Характеристики генерации ДГ при различных установленных елкостях $А Б$

Fig. 13. Characteristics of DG generation at various installed capacity of $S B$

\section{Обсуждение полученных результатов}

Полученные результаты показывают, что в зависимости от состава генерирующего оборудования и установленной емкости АБ существенно изменяются численные значения расчетных коэффициентов и оценок, отвечающих за номер полученной категории и как следствие потенциально возможных технических решений при данных условиях эксплуатации.

АСЭС № 1 - Нол. Исходя из расчетных значений коэффициентов и оценок можно отметить, что с увеличением установленной емкости АБ существенно снижаются численные значения как временных коэффициентов низкого заряда и полного заряда АБ $\left(K_{\mathrm{AB}}^{B-\text { min }}, K_{\mathrm{AB}}^{B \text {-max }}\right)$, так и коэффициент лаксилальной скорости разряда $\left(K_{\mathrm{AB}}^{\text {P-max }}\right)$.

Полученные оценки, характеризующие режимы работы АБ, начиная с установленной емкости от 144 до 432 кВт•ч, соответствуют 1 категории, которая сопровождается тяжелой/необратилой сульфацией, выпадением и разрушением активной массы, а также кислотной стратификацией. При установленной емкости АБ 480 кВт·ч и более коэффициент пропускной способности уменьшается до среднего уровня, что соответствует 6 категории, которая по своей специфике является начальным приближением 1 категории.

АСЭС № 2 - Кодиак. Результат категоризации $A Б$ показывает, что на протяжении всего цикла расчетов режимы работы соответствуют 1 категоpuи. На полученный результат в первую очередь влияют режимы работы элементов генерации, а именно ФЭП, которые имеют ярко выраженный цикличный характер. В таких условиях численные значения коэффициентов $K_{\mathrm{AB}}^{\Pi}, K_{\mathrm{AB}}^{p \text {-max }}, K_{\mathrm{AB}}^{B \text {-max }}, K_{\mathrm{AB}}^{\amalg 3}$ имеют достаточно высокий уровень, что, несомненно, увеличивает требования к выбираемым типам АБ для данных условий эксплуатации.

АСЭС № 3 - АӘак. В целом полученные результаты показывают, что процесс категоризации АБ при совместном использовании энергии ветра и Солнца совпадает с результатами, полученными

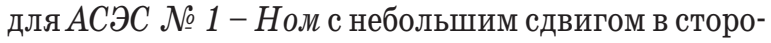


ну увеличения установленной емкости АБ для 1 категории. Следовательно, анализ природноклиматических показателей для н.п. Адак показывает, что скорость ветра на высоте установки лопастей ветрогенератора (30 м) имеет среднемесячные значения 9-11 м/с в зимне-осенний и 7-8 м/с в весенне-летний периоды, что позволяет сделать вывод о главенствующей роли ВЭУ в данном примере.

Таким образом, на основании полученных категорий АБ для АСЭС № 1 - Нол и АСЭС № 3 $A \partial а \kappa$ в пределах суммарной установленной емкости АБ от 144 до 432 и 480 кВт•ч предлагается использовать свиниово-карбоновые АБ, в которых существенно снижается влияние негативных воздействий, присущих 1 категории. Кроме того, для данной категории можно использовать АБ с трубчатыли $(O P z S, O P z V)$ электродами и наполнением как жидкил, так и гелевыл электролитом. При использовании жидкого электролита настоятельно рекомендуется использовать систелу ииркуляиии электролита. При увеличении установленной емкости АБ выше 432 и 480 кВт•ч, предлагается использовать только технологии $O P z S$ и $O P z V$. Для $А С Э С ~ № ~ 2-$ Кодиак рекомендуется использовать так называемые «тяжелые» аккумулирующие устройства, к которым относятся тип свиниово-карбоновых АБ. Как отмечалось ранее, основные процессы деградации АБ, такие как тяжелая/необратилая сульфаиия, выпадение и разрушение активной массы из пластин, а также кислотная стратификаиия, снижаются более чем на $75 \%$. Также одним из возможных вариантов компоновки аккумулирующего звена являются $2 е-$ левые АБ типа $O P z V$.

\section{Выводы}

Результаты, полученные при выполнении данного исследования, демонстрируют состоятельность предложенной методики, направленной на интеграцию процесса категоризации электрохимических накопителей энергии при решении оптимизации состава оборудования АСЭС, использующих ВИЭ.

По результатам исследования были получены численные значения коэффициентов $K_{\mathrm{AБ}}^{3}, K_{\mathrm{AБ}}^{\Pi}$, $K_{\mathrm{Ab}}^{P \text {-max }}, K_{\mathrm{Ab}}^{B-\max }, K_{\mathrm{Ab}}^{ц 3}, K_{\mathrm{AB}}^{B-\min }$, необходимых для опреде-

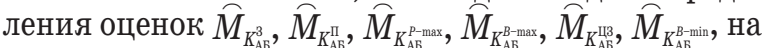
основании которых определяется номер категории АБ. Анализ полученных численных значений оценок в зависимости от суммарной установленной емкости показывает, что происходит изменение технических требований к АБ. Данное положение

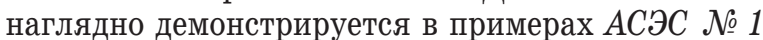
- Нол и АСЭС № 3 - Адак. Следовательно, использование предложенной в рамках данной статьи методики позволяет определить такие типы АБ и технические решения, которые позволят снизить негативные воздействия на внутренние элементы и составные части АБ.

Стоит отметить, что в рамках данного исследования автор статьи с первую очередь акцентировал внимание на процессе моделирования режимных показателей АБ относительно каждого временного интервала $(t)$ с последующим определением численных значений расчетных коэффициентов и оценок, отвечающих за конечный результат категоризации АБ. Таким образом, дальнейшее решение задачи оптимизации состава оборудования может производиться на основании полученных ранее обоснованных технических решений с определением экономической эффективности и выбором соответствующего варианта компоновки.

Кроме того, состоятельность предложенной методики подтверждается тем, что полученные численные результаты категоризации АБ подтверждаются данными с реальных АСЭС с ВИЭ и АБ [23]. Режимные показатели реальных комплексов генерации, использующих ФЭП-ДГ-АБ, в большинстве случаев относятся к 1 категории, что подтверждается примером $A C Э C$ № $2-$ Кодиак. В то же время использование ВЭУ (в т. ч. ВЭУ-ФЭП) может существенно влиять на процесс категоризации в зависимости от суммарной установленной емкости АБ (табл. 3,7 ).

Следует отметить, что данные о минимально допустимом заряде АБ $Q_{\mathrm{Ab}}^{\min }$ принимались по спецификации завода изготовителя (20\% от установленной емкости для типов $O P z S$ и $O P z V$ u $30 \%$ для свиниово-карбоновых АБ). Эти соотношения могут существенно варьироваться, что, несомненно, отразится на частоте включения резервного источника (ДГ), а численные значения коэффициентов $K_{\mathrm{AB}}^{3}, K_{\mathrm{AB}}^{\Pi}, K_{\mathrm{AB}}^{p-\max }, K_{\mathrm{AB}}^{B \text {-max }}, K_{\mathrm{AB}}^{\amalg 3}, K_{\mathrm{AB}}^{B-\min }$, необходимых для определения оценок $\widehat{M}_{K_{A \mathrm{~B}}^{3}}, \widehat{M}_{K_{\mathrm{AB}}^{\Pi}}, \widehat{M}_{K_{\mathrm{AB}}^{p-\max }}, \widehat{M}_{K_{\mathrm{AB}}^{B-\max }}, \widehat{M}_{K_{A \mathrm{~b}}^{\amalg 3}}$, $M_{K^{B-m i n}}$, будут изменяться с учетом данных условий.

Такое положение является отдельным достаточно сложным исследованием, не входящим в рамки текущей статьи.

Стоит отметить, что точность полученных результатов напрямую зависит от уровня детализации используемых математических моделей ACЭС, анализа параметров окружающей среды, peжимных показателей рассматриваемой системы и т. д.

\section{Заключение}

Главная цель статьи направлена на повышение качества решения задачи оптимизации состава оборудования АСЭС, использующих ВИЭ и АБ. Представлен подробный обзор основных задач, возникающих при нахождении оптимального состава оборудования. Произведен графоаналитический анализ структуры АСЭС, где полученный результат позволяет сделать вывод о важном значении АБ, выступающих в роли стабилизирующего элемента автономной энергетической системы.

Представлено математическое описание знакопеременной функиии мощности системы $P_{S}(t)$ с разбивкой на положительную $P_{S}(t)^{+}$и отрицательную $P_{S}(t)^{-}$зоны, характеризующие процессы заряда и разряда АБ относительно каждого временного интервала $(t)$ с соблюдением ряда эксплуатационно-технических ограничений $\bar{a}_{\mathrm{AБ}}$. Описаны основ- 
ные процессы деградации активной массы АБ, возникающие в различных типах электрохимических накопителей энергии. Представлен процесс категоризации АБ в зависимости от условий эксплуатации с описанием основных расчетных коэффициентов с последующим построением шести типовых диаграмм категорий АБ.

Предложена методика интеграции процесса категоризации АБ в задачи оптимизации состава оборудования, использующего ВИЭ и АБ. В рамках данной методики было предложено моделировать АСЭС для условий типичной АБ с определением основных режимных показателей системы для всего периода лет метеорологических наблюдений с последующим определением численных значений расчетных оценок $\widehat{M}_{K_{A \mathrm{~B}}^{3}}, \widehat{M}_{K_{A \mathrm{I}}^{\mathrm{n}}}, \widehat{M}_{K_{A \mathrm{~B}}^{p-\max }}, \widehat{M}_{K_{\mathrm{E}}^{B-\max }}$, $\widehat{M}_{K_{A 5}^{\amalg 13}}, \widehat{M}_{K_{A B}^{B-\min }}$, отвечающих за номер категории АБ.

В ходе работы были исследованы три АСЭС, расположенные в Американском штате Аляска, с различными составами генерирующего оборудования, где на основании процесса категоризации АБ были получены численные значения категорий, что в дальнейшем позволило сформировать матрицу расслатриваелого оборудования. Таким образом, на основании полученной категории выбираются такие технические решения и типы АБ, в которых процессы деградации активной массы сведены к минимальным значениям. В дальнейшем полученные технические решения и типы электрохимических накопителей энергии используются для определения показателя целевой функции $L C O E$ (руб/кВт•ч), где минимальное значение норлированной стоилости электрической энергии будет являться оптимальным решением при данных условиях. При этом автор статьи уверен, что дополнительно необходимо учитывать количе-

\section{СПИСОК ЛИТЕРАТУРЫ}

1. «PA0 ЕЭС Востока». URL: http://www.rao-esv.ru/map (дата обращения: 25.05.2018)

2. Cristóbal-Monreal I.R., Dufo-López R. Optimisation of photovoltaic-diesel-battery stand-alone systems minimising system weight // Energy Conversion and Management. - 2014. - V. 29. P. 151-157.

3. Li F.F., Qiu J. Multi-objective optimization for integrated hydro-photovoltaic power system // Applied Energy. - 2016. V. $167 .-$ P. $377-384$.

4. Оценка целесообразности применения фотоэлектрических установок для электроснабжения удаленных потребителей в климатических условиях Севера Российской Федерации / М.А. Сурков, С.Г. Обухов, И.А. Плотников, Л.П. Сумарокова, М.М. Попов, С.А. Байдали // Интернет-журнал «Науковедение». - 2016. - № 4. - С. 1-13. URL: https://naukovedenie.ru/PDF/97TVN416.pdf (дата обращения: 25.05.2018).

5. Саврасов Ф.В., Лукутин Б.В. Расчёт эффективности использования автономных систем электроснабжения с фотоэлектростанциями в условиях Западной Сибири // Известия Томского политехнического университета. - 2013. - Т. 322. - № 6. C. 17-21.

6. Merei G., Berger C., Sauer D.U. Optimization of an off-grid hybrid PV-Wind-Diesel system with different battery technologies using genetic algorithm // Solar Energy. - 2013. - V. 97. P. $460-473$. ство замен АБ в течение всего срока эксплуатации исходя из их пропускной способности, количества циклов заряд/разряд и предельного количества циклов с учетом минимально допустимого состояния заряда АБ.

\section{Реализация на практике}

Описанная в рамках данной статьи методика применялась при реализации солнечно-дизельного комплекса генерации с АБ «Верхняя Алга», расположенного в Алданском районе Республики Саха (Якутия). По итогам решения задачи оптимизации состава оборудования с учетом процесса категоризаиии было получено, что в рамках данного проекта необходимо использовать свиниово-карбоновые АБ. В октябре 2016 г. станция была введена в эксплуатацию со следующими значениями установленной мощности: ФЭП - 36 кВт, сетевой инвертер - 36 кВт, батарейный инвертер - 24 кВт, свинцово-карбоновые АБ - 144 кВт॰ч.

\section{Дальнейшие исследования}

В первую очередь автор статьи направит свои силы на исследование влияния неопределенности нагрузочной характеристики потребителя на процесс категоризации АБ. Кроме того, автор статьи отмечает, что представленная методика требует дополнительного учета количества замен АБ в течение всего срока эксплуатации, что, несомненно, повлияет на результат оптимизации состава оборудования.

Исследование выполнено в рамках проекта СО РАН: III.17.1.1. Системный анализ влияния показателей технологических процессов и конструкиионных материалов на характеристики перспективных энергетических установок рег. № AAAA-A17-117030310433-6.

7. Modeling and optimization of batteryless hybrid PV (photovoltaic)/Diesel systems for off-grid applications / D. Tsuanyo, Y. Azoumah, D. Aussel, P. Neveu // Energy. - 2015. - V. 86. P. $152-163$

8. Optimization of a Multi-source System with Renewable Energy Based on Ontology / D. Saba, F.Z. Laallam, A.E. Hadidi, B. Berbaoui // Energy Procedia. - 2015. - V. 74. - P. 608-615.

9. Sizing and simulation of a photovoltaic-wind energy system using batteries, applied for a small rural property located in the south of Brazil / C.E.C. Nogueira, M.L. Vidotto, R.K. Niedzialkoski, S.N. Melegari de Souza, L.I. Chaves, T. Edwiges, D. Bentes dos Santos, I. Werncke // Renewable and Sustainable Energy Reviews. - 2014. - V. 29. - P. 151-157.

10. Optimal sizing of a hybrid grid-connected photovoltaic and wind power system / A. González, J-R. Riba, A. Rius, R. Puig // Applied Energy. - 2015. - V. 154. - P. 752-762.

11. Bala B.K., Siddique S. A. Optimal design of a PV-diesel hybrid system for electrification of an isolated island - Sandwip in Bangladesh using genetic algorithm // Energy for Sustainable Development. - 2009. - V. 13. - P. 137-142.

12. Карамов Д.Н. Математическое моделирование автономной системы электроснабжения, использующей возобновляемые источники энергии // Вестник Иркутского государственного технического университета. - 2015. - Т. 104. - № 9. - С. 133-140.

13. Methodology to Size an Optimal Stand-Alone PV/wind/diesel/ battery System Minimizing the Levelized cost of Energy and the 
Emissions / B.O. Bilal, V. Sambou, C.M.F. Kebe, P.A. Ndiaye, M. Ndongo / Energy Procedia. - 2014. - V. 195. - P. 1636-1647.

14. Branker K., Pathak M.J.M., Pearce J.M. A review of solar photovoltaic levelized cost of electricity // Renewable and Sustainable Energy Reviews. - 2011. - V. 15. - P. 4470-4482.

15. Calculation of levelized costs of electricity for various electrical energy storage systems / M. Obi, S.M. Jensen, J.B. Ferris, R.B. Bass // Renewable and Sustainable Energy Reviews. 2017. - V. 67. - P. 908-920.

16. Карамов Д.Н. Оптимизация состава оборудования автономных энергетических комплексов, использующих возобновляемые источники и накопители энергии: дис. ... канд. техн. наук. Иркутск, 2016. - 152 c.

17. Marchenko 0.V., Solomin S.V. Modeling of hydrogen and electrical energy storages in wind/PV energy system on the Lake Baikal coast // International Journal of Hydrogen Energy. - 2017. V. 42. - P. 9361-9370.

18. Marchenko 0.V. Mathematical modeling and economic efficiency assessment of autonomous energy systems with production and storage of secondary energy carriers // International Journal of Low-Carbon Technologies. - 2010. - V. 5. - P. 250-255.

19. Dufo-López R. Optimisation of size and control of grid-connected storage under real time electricity pricing conditions // Applied Energy. - 2015. - V. 140. - P. 395-408.

20. Dufo-López R., Cristóbal-Monreal I.R., Yusta J.M. Optimisation of PV-wind-diesel-battery stand-alone systems to minimise cost and maximise human development index and job creation // Renewable Energy. - 2016. - V. 84. - P. 280-293.

21. Dufo-López R., Lujano-Rojas J.M., Bernal-Agustín J.L. Comparison of different lead-acid battery lifetime prediction models for use in simulation of stand-alone photovoltaic systems // Applied Energy. - 2014. - V. 115. - P. 242-253.

22. Schiffer J., Sauer D.U., Bindner H., Cronin T., Lundsager P., Kaiser R. Model prediction for ranking lead-acid batteries according to expected lifetime in renewable energy systems and autonomous power-supply systems // Journal of Power Sources. - 2007. V. $168 .-$ P. $66-78$.

23. Operating conditions of batteries in off-grid renewable energy systems / V. Svoboda, H. Wenzl, R. Kaiser, A. Jossen, I. BaringGould, J. Manwell, P. Lundsager, H. Bindner, T. Cronin, P. Nørgard, A. Ruddell, A. Perujo, K. Douglas, C. Rodrigues, A. Joyce, S. Tselepis, N. van der Borg, F. Nieuwenhout, N. Wilmot, F. Mattera, D.U. Sauer // Solar Energy. - 2007. - V. 81. P. $1409-1425$.
24. Воропай Н.И. Теория систем для электроэнергетиков. - Новосибирск: Наука, Сибирская издательская фирма РАН, 2000. $273 \mathrm{c.}$

25. Лукутин Б.В., Лукутин О.Б., Шандарова Е.Б. Энергоэффективные системы генерирования электроэнергии для автономных ветроэлектростанций // Известия Томского политехнического университета. - 2005. - Т. 308. - № 7. - С. 203-206.

26. Обухов С.Г., Плотников И.А. Имитационная модель режимов работы автономной фотоэлектрической станции с учетом реальных условий эксплуатации // Известия томского политехнического университета. Инжиниринг георесурсов. - 2017. T. 328. - № 6. - C. 38-51.

27. Применение буферных накопителей энергии для повышения энергоэффективности ветродизельных электростанций / Б.В. Лукутин, С.Г. Обухов, Е.А. Шутов, З.П. Хошнау // Электричество. - 2012. - № 6. - С. 24-29.

28. Dursun E., Kilic 0. Comparative evaluation of different power management strategies of a stand-alone PV/Wind/PEMFC hybrid power system // International Journal of Electrical Power \& Energy Systems. - 2012. - V. 34. - P. 81-89.

29. Analysis of the performance parameters of lead/acid batteries in photovoltaic systems / D.U. Sauer, M. Bächler, G. Bopp, W. Höhe, J. Mittermeier, P. Sprau, B. Willer, M. Wollny // Journal of Power Sources. - 1997. - V. 64. - P. 197-201.

30. Sauer D.U., Garche J. Optimum battery design for applications in photovoltaic systems - theoretical considerations // Journal of Power Sources. - 2001. - V. 95. - P. 130-134.

31. Wagner R., Sauer D.U. Charge strategies for valve-regulated lead/acid batteries in solar power applications // Journal of Power Sources. - 2001. - V. 95. - P. 141-152.

32. Moseley P.T., Garche J. Electrochemical Energy Storage for Renewable Sources and Grid Balancing. $1^{\text {st }}$ ed. - Amsterdam: Elsevier, 2014. $-492 p$

33. Карамов Д.Н. Математическое моделирование солнечной радиации с использованием многолетних метеорологических рядов находящихся в открытом доступе // Известия томского политехнического университета. Инжиниринг георесурсов. 2017. - T. 328. - № 6. - С. $28-38$.

34. Карамов Д.Н. Формирование исходных метеорологических массивов с использованием многолетних рядов FM 12 Synop и METAR в системных энергетических исследованиях // Известия томского политехнического университета. Инжиниринг георесурсов. - 2018. - Т. 329. - № 1. - С. 69-88.

Поступила 04.06.2018 г.

\section{Информация об авторах}

Карамов Д.Н., кандидат технических наук, научный сотрудник лаборатории исследования энергетических установок № 71 отдела теплосиловых систем № 70 Института систем энергетики им. Л.А. Мелентьева Сибирского отделения Российской академии наук; доцент Иркутского национального исследовательского технического университета. 


\title{
INTEGRATION OF THE STORAGE BATTERY CATEGORIZATION PROCESS INTO THE TASK OF OPTIMIZING THE EQUIPMENT OF STAND-ALONE ENERGY SYSTEMS WITH RENEWABLE ENERGY SOURCES
}

\author{
Dmitriy N. Karamov 1,2, \\ dmitriy.karamov@mail.ru \\ ${ }^{1}$ Melentiev Energy Systems Institute of Siberian Branch of the Russian Academy of Science, \\ 130, Lermontov street, Irkutsk, 664033, Russia. \\ ${ }^{2}$ Irkutsk National Research Technical University, \\ 83, Lermontov street, Irkutsk, 664074, Russia.
}

This research arises from the need to enhance the accuracy of optimizing the equipment of stand-alone energy systems with renewable energy sources and storage batteries.

Objective. The paper aims to demonstrate the feasibility of integrating the storage battery categorization process into the task of optimizing the equipment of stand-alone energy systems with renewable energy sources, to identify factors affecting the storage battery categorization process and, finally, to summarize and conduct a detailed analysis of the information obtained.

Methods. The theoretical framework for this paper borrows from the systems theory and commonly known mathematical models that make it possible to configure the main operating parameters of stand-alone energy systems with renewable energy sources and storage batteries. Extensive use is made of actinometric, wind energy and many other natural and climatic indicators obtained by processing long-term meteorological series at weather stations such as Nome, Kodiak and Adak (Alaska, USA).

The results. A methodology was suggested to integrate the storage battery categorization process into the task of optimizing the equipment of stand-alone energy systems using renewable energy sources. The paper presents the research outcomes produced by three conditional stand-alone energy systems using renewable sources and storage batteries. The research findings pointed to the relationship between generation equipment and the total storage battery capacity. An analysis of the research findings was made, followed by appropriate conclusions, their visual representation and assessment of their reliability and potential use in optimization-focused research on stand-alone energy systems.

\section{Key words:}

Renewable energy sources, battery storage categorization, battery storage degradation, system studies, optimization.

The research was carried out within the project of SB RAS: III.17.1.1. System analysis of the effect of process flow and engineering material parameters on characteristics of prospecting power plants reg. no. AAA-A17-117030310433-6

\section{REFERENCES}

1. «RAO EES Vostoka» [«RA0 Energy Systems of the East»]. Available at: http://www.rao-esv.ru/map (accessed 25 May 2018).

2. Cristóbal-Monreal I.R., Dufo-López R. Optimisation of photovoltaic-diesel-battery stand-alone systems minimising system weight. Energy Conversion and Management, 2014, vol. 29, pp. 151-157.

3. Li F.F., Qiu J. Multi-objective optimization for integrated hy dro-photovoltaic power system. Applied Energy, 2016, vol. 167, pp. 377-384.

4. Surkov M.A., Obukhov S.G., Plotnikov I.A., Sumarokova L.P., Popov M.M., Baydali S.A. Feasibility assessment of photovoltaic plants for power supply of remote customers in the climatic conditions of Northern Russia. Naukovedenie, 2016, vol. 8, no. 4, pp. 1-13. In Rus. Available at: https://naukovedenie.ru/ PDF/97TVN416.pdf (accessed 25 May 2018).

5. Savrasov F.V., Lukutin B.V. Calculation of the efficiency of the use of autonomous power supply systems with photoelectric stations in the conditions of Western Siberia. Bulletin of the Tomsk Polytechnic University, 2013, vol. 322, no. 6, pp. 17-21. In Rus.

6. Merei G., Berger C., Sauer D.U. Optimization of an off-grid hybrid PV-Wind-Diesel system with different battery technologies using genetic algorithm. Solar Energy, 2013, vol. 97, pp. $460-473$.

7. Tsuanyo D., Azoumah Y., Aussel D., Neveu P. Modeling and optimization of batteryless hybrid PV (photovoltaic)/Diesel systems for off-grid applications. Energy, 2015, vol. 86, pp. 152-163.
8. Saba D., Laallam F.Z., Hadidi A.E., Berbaoui B. Optimization of a Multi-Source System with Renewable Energy Based on Ontology. Energy Procedia, 2015, vol. 74, pp. 608-615.

9. Nogueira C.E.C., Vidotto M.L., Niedzialkoski R.K., De Souza S.N.M., Chaves L.I., Edwiges T., Dos Santos D.B., Werncke I. Sizing and simulation of a photovoltaic-wind energy system using batteries, applied for a small rural property located in the south of Brazil. Renewable and Sustainable Energy Reviews, 2014, vol. 29, pp. 151-157.

10. González A., Riba J.-R., Rius A., Puig R. Optimal sizing of a hybrid grid-connected photovoltaic and wind power system. Applied Energy, 2015, vol. 154, pp. 752-762.

11. Bala B.K., Siddique S. A. Optimal design of a PV-diesel hybrid system for electrification of an isolated island - Sandwip in Bangladesh using genetic algorithm. Energy for Sustainable Development, 2009, vol. 13, pp. 137-142.

12. Karamov D.N. Mathematical modelling of an autonomous power supply system using renewable energy sources. Bulletin of Irkutsk State Technical University, 2015, vol. 104, no. 9, pp. 133-140. In Rus.

13. Bilal B.O., Sambou V., Kebe C.M.F., Ndiaye P.A., Ndongo M. Methodology to Size an Optimal Stand-Alone PV/wind/diesel/battery System Minimizing the Levelized cost of Energy and the $\mathrm{CO}_{2}$ Emissions. Energy Procedia, 2014, vol. 195, pp. 1636-1647.

14. Branker K., Pathak M.J.M., Pearce J.M. A review of solar photovoltaic levelized cost of electricity. Renewable and Sustainable Energy Reviews, 2011, vol. 15, pp. 4470-4482. 
15. Obi M., Jensen S.M., Ferris J. B., Bass R. B. Calculation of levelized costs of electricity for various electrical energy storage systems. Renewable and Sustainable Energy Reviews, 2017, vol. 67, pp. 908-920.

16. Karamov D.N. Optimizasiya sostava oborudovaniya avtonomnykh energeticheskikh kompleksov, ispolzuyushchikh vozobnovly aemye istochniki $i$ nakopiteli energii. Avtoreferat Kand. nauk [Optimization of the equipment composition of autonomous energy complexes using renewable sources and energy storages. Cand. Diss]. Irkutsk, 2016. 152 p.

17. Marchenko 0.V., Solomin S.V. Modeling of hydrogen and electrical energy storages in wind/PV energy system on the Lake Baikal coast. International Journal of Hydrogen Energy, 2017, vol. 42, pp. 9361-9370.

18. Marchenko 0.V. Mathematical modeling and economic efficiency assessment of autonomous energy systems with production and storage of secondary energy carriers. International Journal of Low-Carbon Technologies, 2010, vol. 5, pp. 250-255.

19. Dufo-López R. Optimisation of size and control of grid-connected storage under real time electricity pricing conditions. Applied Energy, 2015, vol. 140, pp. 395-408.

20. Dufo-López R., Cristóbal-Monreal I.R., Yusta J. M. Optimisation of PV-wind-diesel-battery stand-alone systems to minimise cost and maximise human development index and job creation. Renewable Energy, 2016, vol. 84, pp. 280-293.

21. Dufo-López R., Lujano-Rojas J. M., Bernal-Agustín J. L. Comparison of different lead-acid battery lifetime prediction models for use in simulation of stand-alone photovoltaic systems. Applied Energy, 2014, vol. 115, pp. 242-253.

22. Schiffer J., Sauer D. U., Bindner H., Cronin T., Lundsager P., Kaiser R. Model prediction for ranking lead-acid batteries according to expected lifetime in renewable energy systems and autonomous power-supply systems. Journal of Power Sources, 2007, vol. 168 , pp. $66-78$.

23. Svoboda V., Wenzl H., Kaiser R., Jossen A., Baring-Gould I., Manwell J., Lundsager P., Bindner H., Cronin T., Nørgard P., Ruddell A., Perujo A., Douglas K., Rodrigues C., Joyce A., Tselepis S., van der Borg N., Nieuwenhout F., Wilmot N., Mattera F., Sauer D. U. Operating conditions of batteries in off-grid renewable energy systems. Solar Energy, 2007, vol. 81, pp. 1409-1425.

24. Voropai N.I. Teoriya sistem dlya elektroenergetikov [Theory of systems for electric power industry]. Novosibirsk, Nauka Publ., $2000.273 \mathrm{p}$.

\section{Information about the authors}

Dmitriy N. Karamov, Cand. Sc., researcher, Melentiev Energy Systems Institute of Siberian Branch of the Russian Academy of Science; associate professor, Irkutsk National Research Technical University.
25. Lukutin B.V., Lukutin 0.B., Shandarova E.B. Energy-efficient power generation systems for autonomous wind stations. Bulletin of the Tomsk Polytechnic University, 2005, vol. 308, no. 7, pp. 203-206. In Rus.

26. Obukhov S.G., Plotnikov I.A. Simulation model of operation of autonomous photovoltaic plant under actual operating conditions. Bulletin of the Tomsk Polytechnic University. Geo Assets Engineering, 2017, vol. 328, no. 6, pp. 38-51. In Rus.

27. Lukutin B.V., Obukhov S.G., Shutov E.A., Khoshnau Z.P. Primenenie bufernykh nakopiteley energii dlya povysheniya energoeffektivnosti vetrodizelnykh elektrostantsiy [Use of buffer storage for increasing energy efficiency wind-diesel power stations]. Electricity, 2012, no. 6, pp. 24-29.

28. Dursun E., Kilic 0. Comparative evaluation of different power management strategies of a stand-alone PV/Wind/PEMFC hybrid power system. International Journal of Electrical Power \& Energy Systems, 2012, vol. 34, pp. 81-89.

29. Sauer D.U., Bächler M., Bopp G., Höhe W., Mittermeier J., Sprau P., Willer B., Wollny M. Analysis of the performance parameters of lead/acid batteries in photovoltaic systems. Journal of Power Sources, 1997, vol. 64, pp. 197-201.

30. Sauer D.U., Garche J. Optimum battery design for applications in photovoltaic systems - theoretical considerations. Journal of Power Sources, 2001, vol. 95, pp. 130-134.

31. Wagner R., Sauer D.U. Charge strategies for valve-regulated lead/acid batteries in solar power applications. Journal of Power Sources, 2001, vol. 95, pp. 141-152. ewable Sources and Grid Balancing. $1^{\text {st }}$ ed. Amsterdam, Elsevier, 2014. $492 \mathrm{p}$.

33. Karamov D.N. Mathematical modeling of solar radiation based on open access long-term meteorological observation data. Bulletin of the Tomsk Polytechnic University. Geo Assets Engineering, 2017, vol. 328, no. 6, pp. 28-37. In Rus.

34. Karamov D.N. Formation of initial meteorological arrays with the use of long-term series FM 12 Synop and METAR in systems energy studies. Bulletin of the Tomsk Polytechnic University. Geo Assets Engineering, 2018, vol. 329, no. 1. 69-88. In Rus.

Received: 4 June 2018.
32. Moseley P.T., Garch J. Electrochemical Energy Storage for Ren- 